\title{
Localic completion of generalized metric spaces II: Powerlocales
}

\author{
STEVEN VICKERS
}

\begin{abstract}
The work investigates the powerlocales (lower, upper, Vietoris) of localic completions of generalized metric spaces. The main result is that all three are localic completions of generalized metric powerspaces, on the Kuratowski finite powerset. This is a constructive, localic version of spatial results of Bonsangue et al. and of Edalat and Heckmann.
\end{abstract}

As applications, a localic completion is always overt, and is compact iff its generalized metric space is totally bounded.

The representation is used to discuss closed intervals of the reals, with the localic Heine-Borel Theorem as a consequence.

The work is constructive in the topos-valid sense.

2000 Mathematics Subject Classification 54B20 (primary); 06D22, 03G30,54E50,03F60 (secondary)

Keywords: locale, constructive, topos, metric, hyperspace, powerlocale

\section{Introduction}

In the geometric approach to point-free topology, as outlined in some detail in [32], a prominent place in the reasoning style is occupied by the powerlocales (or point-free hyperspaces). There is therefore an intrinsic interest in investigating how powerlocales interact with geometric modes of defining point-free spaces. The present paper studies powerlocales when applied to localic completions [33] of metric spaces (in fact, of generalized metric spaces in the sense of Lawvere [14]), and shows that the powerlocales too are localic completions, got by taking appropriate generalized metrics on the finite powersets of the original spaces. ${ }^{1}$

Hyperspaces from the start have been associated with metric spaces. (From the localic point of view, a convenient account of the historical background is given in [7] in its

\footnotetext{
${ }^{1}$ The core technical content of those results has already appeared in [29].
} 
description of the Vietoris powerlocale. Another interesting summary, from the point of view of non-standard analysis, is in [39].) If $X$ is a metric space, then the Hausdorff metric on the powerset $\mathcal{P} X$ is defined by

$$
d_{H}(A, B)=\max (m(A, B), m(B, A))
$$

where

$$
m(A, B)=\sup _{a \in A} \inf _{b \in B} d(a, b) .
$$

In this generality we have that $m$ and $d_{H}$ satisfy the triangle inequality, that $d_{H}(A, A)=$ $m(A, A)=0$, and that $d_{H}$ (though not $m$ ) is symmetric. However, they are not metrics as they stand. First, $m(A, B)$ may be infinite if $A$ is unbounded or $B$ is empty. Next, $d_{H}(A, \mathrm{Cl}(A))=0$ where $\mathrm{Cl}(A)$ is the closure of $A$-indeed, $m(A, B)=0$ iff $A \subseteq \mathrm{Cl}(B)$. We get a metric space by restricting $d_{H}$ to the closed, bounded, non-empty subspaces of $X$.

Restricting to compact non-empty subspaces, the topology induced by the Hausdorff metric can also be got by purely topological means, from the Vietoris topology on $\mathcal{P} X$. This is given by a subbase of opens, comprising sets of the form

$$
\begin{aligned}
& \square U=\{A \mid A \subseteq U\} \\
& \diamond U=\{A \mid A \cap U \neq \emptyset\}
\end{aligned}
$$

where $U$ is open in $X$. This Vietoris topology motivated Johnstone's construction [9] of what we now call the Vietoris powerlocale. (It is also described in preliminary form - with an unnecessary restriction to the compact regular case - in [7], together with detailed historical notes.) The aim of the present paper is to reconstruct in localic terms the connection between Hausdorff metric and Vietoris topology. We shall see the metric reappearing at the level of finite subsets of the metric space.

It is also natural to ask how the hyperspace theory might appear in the setting of generalizations such as quasimetrics (for which the symmetry axiom is dropped). In fact the whole of the present paper depends on this, since the upper and lower powerspaces are non-symmetric. Another example is the lower reals (Definition 7.5); in fact nonsymmetry goes naturally with non- $T_{1}$ spaces (non-discrete specialization order). It is then fruitful to modify the definition of the Hausdorff metric, so that it is not automatically symmetric. If we take $m$, as defined above, as a lower quasimetric $d_{L}$ on sets, and define an upper quasimetric $d_{U}(A, B)=\sup _{b \in B} \inf _{a \in A} d(a, b)$ (which would equal $m(B, A)$ if $d$ were symmetric), then we can take $d_{H}(A, B)=\max \left(d_{L}(A, B), d_{U}(A, B)\right)$.

Bonsangue et al. [2] have already studied hyperspaces for Lawvere's generalized metric spaces, in which the only assumptions are zero self-distance and the triangle inequality: 
the metric may be asymmetric, may take infinite values, and may fail the axiom that if $d(x, y)=d(y, x)=0$ then $x=y$. In [2] the approach to hyperspaces is adopted from domain theory, which distinguishes between the convex powerdomain, analogous to the Vietoris hyperspace, and two other "lower" and "upper" powerdomains. Given a complete generalized metric space $X$, equipped with what they define as a "basis" $B$, they define the "lower powerdomain" of $X$ as a certain subspace of a continuous dcpo $\widehat{B}$ that can also be understood as the lower powerdomain of a ball domain, a continuous dcpo as developed in [3] and [20]. Having defined this lower powerdomain, [2] shows that it can also be got by completing the finite powerset $\mathcal{F} B$ equipped with a "lower metric" (in effect, one part of the Vietoris metric). The paper also sketches similar results for upper and convex powerdomains. To summarize, hyperspaces (powerdomains; lower, upper or convex) of generalized metric space completions can be got as completions of finite powersets equipped with an appropriate generalized metric (lower, upper or convex).

There are analogous constructions in [3] for the convex powerdomain (Vietoris hyperspace) of an ordinary metric space, and there we see extensive use of the ball domain idea. This embeds the metric space as the maximal points in a continuous dcpo of formal balls, the maximal points being balls of zero radius. The idea seems to originate in the interval domain [21], where the same idea is applied to the reals. Once the embedding has been made, techniques of continuous dcpo theory can be applied to gain results about the metric space.

Our companion paper [33] describes a localic treatment of generalized metric space completion: for each generalized metric space $X$, it defines a locale $\bar{X}$, its "localic completion". The points of $\bar{X}$ are Cauchy filters of formal open balls in $X$. In the symmetric case these are classically equivalent to equivalence classes of Cauchy sequences, and more generally to the elements of the completion described in [2]. However, the descriptions used in [2] and in [33] are radically different. The completion in [2] is a "least subset closed under limits", and even in classical mathematics it is not amenable to localic development - the classical equivalence proof in [33] is rather intricate. From the localic point of view, the completion by Cauchy filters of formal balls is much more satisfactory, essentially because those Cauchy filters are the models of a propositional geometric theory. (One might argue that in any case the construction is simpler than that in [2].) Moreover, the reasoning is easily done constructively - one great advantage of the localic approach to topology is that it gives better constructive results.

The effect of the present paper is to translate the powerdomain results of [2] into the setting of the localic completion. However, their definitions of powerdomains are re- 
placed by the localic constructions of powerlocales, the localic version of hyperspaces, and for this reason the technical development here is almost entirely new and somewhat algebraic in nature. There is still a measure of comparison through our use of ball domains, and in that respect the present paper gives a localic account of a generalization (to generalized metric spaces and to all three powerdomains) of [3]. We shall show that the powerlocales of $\bar{X}$ may themselves also be described as localic completions, with respect to three different generalized metrics on the (Kuratowski) finite powerset $\mathcal{F} X$ of $X$. The Vietoris powerlocale, which is by definition analogous to the Vietoris hyperspace topology, in this situation uses the Hausdorff metric restricted to finite subsets. The lower and upper powerlocales correspond to different topologies with subbases given by the opens $\square U$ and $\diamond U$ respectively. Their corresponding metrics are asymmetric, even for symmetric $X$.

We give two sets of applications. The first exploits the fact that some properties of locales can be expressed as structure existing in the powerlocales. For instance, a locale is compact iff its upper powerlocale is colocal (has, in a certain universal sense, a top point). This then makes it easy to characterize compactness of $\bar{X}$ in terms of a total boundedness property on the generalized metric space $X$. A similar argument with the lower powerlocale yields the result that all completions $\bar{X}$ are overt (open) as locales, a property that is classically trivial but constructively important.

The second set of applications is to the real line $\mathbb{R}$, as completion $\overline{\mathbb{Q}}$ of the rationals. Our techniques make it easy to define the closed interval $[0,1]$ as a point of the Vietoris powerlocale $V \mathbb{R}$, and then its compactness (the Heine-Borel Theorem) follows immediately from the way points of $V X$ (for any locale $X$ ) correspond to certain compact sublocales of $X$. The localic Heine-Borel Theorem is, of course, known already [4], and is a good example of how in constructive mathematics, ordinary topology works better with a point-free approach. With the powerlocales we can strengthen this by defining the interval $[x, y]$ for general $x \leq y$, and indeed we see how $[x, y]$ depends continuously on $x$ and $y$. This is developed further in [38], with an account of the Intermediate Value Theorem.

The paper is constructive throughout in the sense of topos validity. 


\subsection{Outline of development}

We shall be working within a context of categories

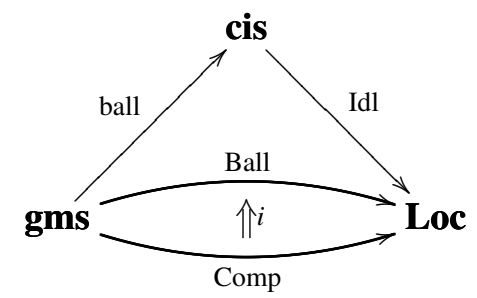

where Loc, gms and cis are the categories of locales, generalized metric spaces and continuous information systems (Sections 2.1, 2.4 and 2.3).

The functor of central interest is the localic completion functor $\operatorname{Comp}(X)=\bar{X}$. Our main result (Theorem 5.4) is that each powerlocale $P_{\sim}$ (lower, upper or Vietoris) applied to a completion $\bar{X}$ can be got by completing an elementary construction based on the finite powerset $\mathcal{F} X$. We find three powerspace constructions $\mathcal{F}_{\sim}$ on gms (Section 3), and natural isomorphisms

$$
v_{\sim}: \operatorname{Comp} \circ \mathcal{F}_{\sim} \cong P_{\sim} \circ \text { Comp } .
$$

While the diagram is in front of us, let us mention that in our context Comp is not an endofunctor and does not lead to a completion monad over which some power monad might distribute. We do not at present have notions of "generalized metric locale", or complete such, and Comp acts between two quite distinct categories.

The rest of the diagram concerns tools used in constructing these isomorphisms. These reduce to the similar, but simpler, results [26] for the ideal completion functor Idl : cis $\rightarrow$ Loc. They involve constructions $\mathcal{F}_{\sim}$ on cis (again based on the finite powerset; Proposition 2.11) and natural isomorphisms (Definition 2.12)

$$
v_{\sim}^{\prime}: \operatorname{Idl} \circ \mathcal{F}_{\sim} \cong P_{\sim} \circ \mathrm{Idl} .
$$

These are combined using the ball domain idea (Section 4). This can be understood at an elementary level as a functor ball: gms $\rightarrow$ cis, under which ball $(X)$ is an information system of formal balls ordered by refinement. There are again natural transformations

$$
\phi_{\sim}: \text { ball } \circ \mathcal{F}_{\sim} \rightarrow \mathcal{F}_{\sim} \circ \text { ball }
$$

(Definition 4.4), though this time not isomorphisms. From these we can derive (Definition 4.7)

$$
\phi_{\sim}^{\prime}: \text { Ball } \circ \mathcal{F}_{\sim} \rightarrow P_{\sim} \circ \text { Ball }
$$


in which $\operatorname{Ball}(X)=\operatorname{Idl} \circ \operatorname{ball}(X)$ is the ball domain.

We can take $\phi_{\sim}^{\prime}$ as already understood, since $v_{\sim}^{\prime}$ is known from [26] and $\phi_{\sim}$ is elementary. Our strategy now (Sections 5.1 and 5.2) is to use an embedding $i:$ Comp $\rightarrow$ Ball (Definition 4.3) and show how $v_{\sim}$ can be obtained by restricting $\phi_{\sim}^{\prime}$ and, moreover, that it becomes a natural isomorphism.

An important fact about the powerlocales is that they are the functor parts of monads (in the categorical sense: see [16]) on Loc, so it is useful to know that there is also monad structure on the $\mathcal{F}_{\sim}$ constructions and that Comp and Idl preserve it (modulo the isomorphisms $v_{\sim}$ and $v_{\sim}^{\prime}$ ). This last fact can be expressed technically by saying that (Comp, $v_{\sim}$ ) and (Idl, $v_{\sim}^{\prime}$ ) are monad opfunctors ([23]; see our Section 2.2). That notion does not depend on $v_{\sim}$ and $v_{\sim}^{\prime}$ being isomorphisms, and in fact it will prove useful to know that (ball, $\phi_{\sim}$ ) and (Ball, $\phi_{\sim}^{\prime}$ ) are also monad opfunctors.

\subsection{Notation}

We mention some notational features of the paper that are not entirely standard.

Composition: The default order for composition of functions or morphisms is the "applicative" order, sometimes emphasized with a symbol. Thus if $f: X \rightarrow Y$ and $g: Y \rightarrow Z$ then $g f=g \circ f: X \rightarrow Z$ with $(g \circ f)(x)=g(f(x))$. Occasionally it is convenient to use the diagrammatic order, and for this the ; symbol is adopted from computer programming. Thus $f ; g=g f$. For natural transformations the "vertical" composition, got by composing components, is often written vertically. This is explained and illustrated in Section 2.2.

Down closure etc.: Suppose $R$ is a relation from $A$ to $B$ and $B^{\prime} \subseteq B$. Then $R B^{\prime}$ denotes the inverse image $\left\{a \in A \mid \exists b \in B^{\prime}\right.$. $\left.a R b\right\}$. This is particularly useful in association with orders. If $\leq$ is a partial order on $A$, then $\leq A^{\prime}$ and $\geq A^{\prime}$ are the down closure and up closure of $A^{\prime}$. Similarly for a non-reflexive order $<$ we might write $<A^{\prime}$ and $>A^{\prime}$, though these will no longer include $A^{\prime}$ and so are not closures.

\section{Background}

\subsection{Locales and powerlocales}

For the general background on locales, see [7] or [25]. We shall use extensively the technique that locales and locale maps can be described pointwise, so long as 
the description is geometric: the points are described as the models of a geometric theory, and a map is described using constructions of geometric constructivism, ie those constructions that are preserved by the inverse image parts of geometric morphisms. Principally, those are colimits, finite limits and free algebra constructions for finitary theories - including the finite powerset, as free semilattice. This is explained rather more carefully in [38]. For technical detail see [32], which also explains how locale constructions (notably powerlocales) may be geometric, as well as other papers such as [36], [30] and [31].

A propositional geometric theory is presented by a set $G$ of propositional symbols, and a set $R$ of axioms of the form $\phi \rightarrow \psi$ where $\phi$ and $\psi$ are geometric formulae: they are built from the propositional symbols using finitary conjunction and arbitrary (possibly infinitary) disjunction. But this is formally equivalent to a presentation $\operatorname{Fr}\langle G \mid R\rangle$ of a frame by generators $G$ and relations $R$, each axiom $\phi \rightarrow \psi$ being interpreted as a relation $\phi \leq \psi$, with conjunction and disjunction interpreted as frame theoretic meet and join - this frame is the (geometric) Lindenbaum algebra of the theory. Algebraically, its universal property is that for any frame $A$ and function $f: G \rightarrow A$ respecting the relations (they become true in $A$ ), there is a unique frame homomorphism $f^{\prime}: \operatorname{Fr}\langle G \mid R\rangle \rightarrow A$ that agrees with $f$ on the generators. If $G$ itself has structure of a kind that exists in frames (for example poset, semilattice, lattice), we often write "(qua ... that kind of structure)" to indicate implicit relations requiring the structure of $G$ to be preserved in the frame presented.

The Vietoris powerlocale was introduced in [9]; see also [7]. The upper and lower powerlocales were in effect derived from it, influenced by the parallel development of three powerdomains in the denotational semantics of computer programs. The localic connection was made in [40] and [19]. For further remarks on their history see [28], whose technical development we shall largely follow (see also [27]).

Definition 2.1 As in [13], a suplattice is a complete join semilattice. A suplattice homomorphism preserves all joins.

As in [12] (and following Banaschewski), a preframe is a poset with finite meets and also directed joins, over which the binary meet distributes. A preframe homomorphism preserves the finite meets and directed joins.

Definition 2.2 If $X$ is a locale, then the lower and upper powerlocales $P_{L} X$ and $P_{U} X$ are defined by letting $\Omega P_{L} X$ and $\Omega P_{U} X$ be the frames generated freely over $\Omega X$ qua suplattice and qua preframe respectively, with generators written as $\diamond a$ and $\square a$ $(a \in \Omega X)$. "Qua suplattice" and "qua preframe" say that $\diamond$ preserves joins and 
preserves finite meets and directed joins. Then if $A$ is any frame and $f: \Omega X \rightarrow A$ is a suplattice homomorphism, then there is a unique frame homomorphism $f^{\prime}: \Omega P_{L} X \rightarrow A$ such that $f^{\prime}(\nabla a)=f(a)$, and similarly for preframe homomorphisms and $P_{U} X$.

The Vietoris powerlocale $V X$ is the "Vietoris construction" of [9]. It is the sublocale of $P_{L} X \times P_{U} X$ presented by relations

$$
\begin{aligned}
\nabla a \times \square b & \leq \diamond(a \wedge b) \times P_{U} X \\
P_{L} X \times \square(a \vee b) & \leq \nabla a \odot \square b .
\end{aligned}
$$

(In a product locale $U \times V$, with $a \in \Omega U$ and $b \in \Omega V, a \odot b$ denotes $a \times V \vee U \times b$.) We shall frequently write $V X$ as $P_{C} X$ to facilitate general arguments that apply to $P_{\sim} X$ where $\sim$ can stand for L, U or C. C here stands for "convex", but this is in a sense of order theory rather than geometry. This is further explained after Theorem 2.3.

The three powerlocales all have positive parts. $P_{L}^{+} X$ is the open sublocale $\diamond X, P_{U}^{+} X$ is the closed sublocale $P_{U} X-\square \emptyset$, and $V^{+} X$ is the restriction of $V X$ to $P_{L}^{+} X \times P_{U}^{+} X$.

The term "powerlocale" is explained by the fact that their points can be considered as sublocales. The results, all topos-valid, are surveyed in [28]. Sublocales (the localic notion of subspace) are described in the standard texts, but see also [37] for a survey from the geometric point of view.

The global points of $P_{U} X$ are easily seen to be equivalent to Scott open filters of $\Omega X$, and a localic form (essentially due to Johnstone [9], though the constructive proof [28] is somewhat different) of the Hofmann-Mislove Theorem ([6]; see also [25]) shows that these are exactly the open neighbourhood filters of the compact, fitted sublocales of $X$. (A sublocale is fitted iff it is the sublocale meet of its open neighbourhoods.) Note that the specialization order in $P_{U} X$ is the opposite of sublocale inclusion: a large Scott open filter corresponds to a small sublocale. $\emptyset$ is the top point in $P_{U} X$, the Scott open filter containing all the opens of $X$.

The global points of $P_{L} X$ are equivalent to the completely prime upsets $F$ in $\Omega X$. Classically, by taking the closed complement of the join of the opens not in $F$, one finds that these are equivalent to closed sublocales of $X$. The constructive version is slightly more complicated, and the completely prime upsets are equivalent to overt, weakly closed sublocales of $X$. (Overtness of locales will be discussed in more detail in Section 6.2. A sublocale of $X$ is weakly closed iff it is a meet of sublocales of the form $(X-U) \vee !^{*} p$, where $U$ is open and $p$ is a proposition (truth value). !: $X \rightarrow 1$ is the unique map, and so $!^{*} p$ denotes the sublocale $\bigvee\{X \mid p\}$.) The completely prime upset corresponding to an overt, weakly closed sublocale $K$ comprises the opens of $X$ 
that are positive modulo $K$. The specialization order for $P_{L} X$ agrees with the inclusion order for sublocales: a large sublocale has more positive opens. $\emptyset$ is the bottom point in $P_{L} X$.

The global points of $V X$ are equivalent to the compact, overt, weakly semifitted sublocales of $X$, where a sublocale is weakly semifitted if it is a meet of a fitted sublocale and a weakly closed sublocale.

There are embeddings $\uparrow: X \rightarrow P_{U}^{+} X, \downarrow: X \rightarrow P_{L}^{+} X$ and $\{-\}: X \rightarrow V^{+} X$, whose inverse image functions take (as appropriate) $\square a$ to $a$ and $\diamond a$ to $a$. For a point $x$ of $X, \uparrow x$ is the sublocale comprising those points $y$ with $x \sqsubseteq y$, and $\downarrow x$ comprises those with $y \sqsubseteq x .\{x\}$ comprises the point $x$ only. We vary this notation by writing $\Downarrow: V X \rightarrow P_{L} X$ and $\Uparrow: V X \rightarrow P_{U} X$ for the projection maps restricted to $V X$.

Theorem 2.3 [27] Let $X$ be a locale, and let $K$ be a point of $P \sim X$ where $\sim$ is $U, L$ or $C$. Then the corresponding sublocale of $X$ comprises those points $x$ such that (for the three cases of $\sim$ ) -

$$
\begin{aligned}
& U: \quad x \equiv K, \\
& L: \quad \downarrow x \sqsubseteq K, \text { or } \\
& C: \quad \uparrow x \sqsupseteq \Uparrow K \text { and } \downarrow x \sqsubseteq \Downarrow K .
\end{aligned}
$$

Note that a sublocale $K$ corresponding to a point of the upper powerlocale $P_{U} X$ is upper closed with respect to the specialization order: if $x$ is in $K$ and $x \sqsubseteq y$ then $y$ is in $K$. (This is an immediate consequence of the fact that $K$ is fitted.) Similarly, for $P_{L} X$ the sublocales are lower closed. For $V X$ (also known as $P_{C} X$ ), the sublocales are order convex - if $x \sqsubseteq y \sqsubseteq z$ with $x$ and $z$ both in $K$, then $y$ is in $K$.

\subsection{Monads and monad opfunctors}

An important aspect of all three powerlocales, as well as their three positive parts, is that they are the functor parts of monads on the category Loc of locales. The units are $\uparrow$, $\downarrow$ and $\{-\}$. The multiplications are analogues of the union function $\bigcup: \mathcal{P} \mathcal{P} X \rightarrow \mathcal{P} X$ and, following [27], we write them as $\sqcap: P_{U}^{2} \rightarrow P_{U}, \sqcup: P_{L}^{2} \rightarrow P_{L}$ and $\cup: V^{2} \rightarrow V$. Their inverse image functions take (as appropriate) $\square a$ to $\square \square a$ and $\diamond a$ to $\diamond \diamond a$. In this section we summarize relevant parts of the abstract theory of monads.

The notion of monad itself is familiar from [16], but let us repeat the definition here. 
Definition 2.4 Let $\mathcal{C}$ be a category. A monad on $\mathcal{C}$ is an endofunctor $T: \mathcal{C} \rightarrow \mathcal{C}$ equipped with two natural transformations $\eta: \mathcal{C} \rightarrow T$ and $\mu: T^{2} \rightarrow T$, satisfying the equations

$$
\frac{T \mid \eta}{\mu}=\frac{\eta \mid T}{\mu}=T \text { and } \frac{\mu \mid T}{\mu}=\frac{T \mid \mu}{\mu} .
$$

There are some notational conventions here that we shall use throughout. First, we use the name of a category $\mathcal{C}$ also for the identity functor $\operatorname{Id}_{\mathcal{C}}$ on it (as in $\eta: \mathcal{C} \rightarrow T$ ), and the name of a functor for the identity natural transformation on it (as for both instances of $T$ in $\begin{gathered}\eta T \\ \mu\end{gathered}=T$ ). Second, we write horizontal composition of natural transformations horizontally, with the domain on the right, and vertical composition vertically, with the domain at the top. In displayed equations we shall mark these with vertical and horizontal lines, aligned to give an indication of how the domains and codomains match up.

As an example, consider the equation $\begin{gathered}\mu T \\ \mu\end{gathered}=\begin{gathered}T \mu \\ \mu\end{gathered}$. The horizontal composite $\mu T: T^{3} \rightarrow T^{2}$ is got by taking components of $\mu$ at objects $T X$, while $T \mu: T^{3} \rightarrow T^{2}$ is got by applying $T$ to components of $\mu$. Now bearing in mind that vertical composition is got by composing components of natural transformations, we see that the equation stands for a commutative diagram as follows (for all $X$ ).

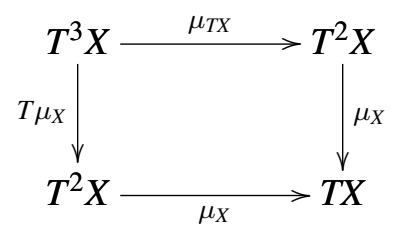

Example 2.5 The best known examples of monads on Set are those in which $T X$ is a free algebra over $X$ for some given algebraic theory. One that is related to the powerlocales is the free semilattice, given concretely by the finite powerset $\mathcal{F} X$ with $\cup$ as its semilattice operation. (Similarly, the full powerset $\mathcal{P} X$ is the free suplattice.) Functorially, if $f: X \rightarrow Y$ then $\mathcal{F} f$ gives direct images. The unit of the monad is the singleton map $\{-\}: X \rightarrow \mathcal{F} X, x \mapsto\{x\}$, and the multiplication is the union map $\bigcup: \mathcal{F F} X \rightarrow \mathcal{F} X$.

"Finite" here means Kuratowski finite: a set is finite if its elements can be listed in a finite list, possibly with repetitions. Note that emptiness of finite sets is a decidable property. We write $\mathcal{F}^{+} X$ for the set of non-empty finite subsets of $X$. The monad structure on $\mathcal{F} X$ restricts to one on $\mathcal{F}^{+} X$. 
We now turn to the monad opfunctors. Street [23] defines, for any 2-category $\mathbb{C}$, a 2-category Mnd( $(\mathbb{C})$ whose 0-cells, 1-cells and 2-cells are monads, monad functors and monad functor transformations. The paper also introduces dual terminology corresponding to $\operatorname{Mnd}\left(\mathbb{C}^{*}\right)^{*}$, where $\mathbb{C}^{*}$ is the dual got by reversing 1-cells, and in fact those monad opfunctors will be more relevant to us here. Specializing to the case $\mathbb{C}=$ Cat we have the following definitions.

Definition 2.6 Suppose $\mathcal{C}_{i}(i=1,2)$ are categories, with monads $\left(T_{i}, \eta_{i}, \mu_{i}\right)$. A monad opfunctor from $\left(\mathcal{C}_{1}, T_{1}\right)$ to $\left(\mathcal{C}_{2}, T_{2}\right)$ is a pair $(F, \phi)$ where $F: \mathcal{C}_{1} \rightarrow \mathcal{C}_{2}$ is a functor and $\phi: F T_{1} \rightarrow T_{2} F$ is a natural transformation such that

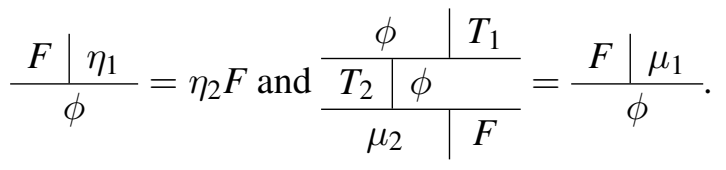

Given a second monad opfunctor $(G, \psi):\left(\mathcal{C}_{2}, T_{2}\right) \rightarrow\left(\mathcal{C}_{3}, T_{3}\right)$, their composite is $\left(G F, \begin{array}{c}G \phi \\ \psi F\end{array}\right):\left(\mathcal{C}_{1}, T_{1}\right) \rightarrow\left(\mathcal{C}_{3}, T_{3}\right)$.

If $(F, \phi)$ and $\left(F^{\prime}, \phi^{\prime}\right)$ are two monad opfunctors from $\left(\mathcal{C}_{1}, T_{1}\right)$ to $\left(\mathcal{C}_{2}, T_{2}\right)$, then a monad opfunctor transformation from $(F, \phi)$ to $\left(F^{\prime}, \phi^{\prime}\right)$ is a natural transformation $\alpha: F \rightarrow F^{\prime}$ such that $\begin{gathered}\alpha T_{1} \\ \phi^{\prime}\end{gathered}=\begin{gathered}\phi \\ T_{2} \alpha\end{gathered}$.

The dual terminology is similar. A monad functor from $\left(\mathcal{C}_{1}, T_{1}\right)$ to $\left(\mathcal{C}_{2}, T_{2}\right)$ is a pair $(F, \psi)$ where $F: \mathcal{C}_{1} \rightarrow \mathcal{C}_{2}$ is a functor and $\psi: T_{2} F \rightarrow F T_{1}$ is a natural transformation such that the corresponding dual equations hold. Transformations of monad functors are defined similarly to those for monad opfunctors.

If $\phi$ is a natural isomorphism, then $(F, \phi)$ is a monad opfunctor iff $\left(F, \phi^{-1}\right)$ is a monad functor and these are equivalent ways of saying that $F$ preserves the monad structure modulo the isomorphism. Our main result (Theorem 5.4) is that localic completion does this, with respect to the powerspace and powerlocale monads, and as such it could be stated in terms of either monad functors or monad opfunctors. However, as mentioned already in Section 1.1, along the way we find ourselves using transformations $\phi$ that are not isomorphisms but still give monad opfunctors. Hence we see monad opfunctors as a kind of symmetry breaking of the main idea of preserving monad structure modulo isomorphism.

As an exercise, the reader unfamiliar with the 2-dimensional calculus might like to verify that the equation $\begin{gathered}\phi T_{1} \\ T_{2} \phi \\ \mu_{2} F\end{gathered}=\begin{gathered}F \mu_{1} \\ \phi\end{gathered}$ above stands for a commutative diagram as 
follows (for all $X$ ).

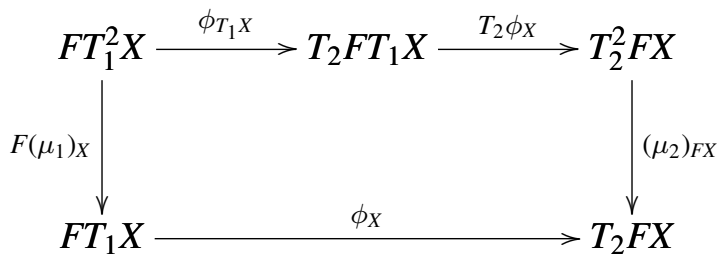

Next we prove some abstract lemmas that will be useful later.

Lemma 2.7 Let $(F, \phi):\left(\mathcal{C}_{1}, T_{1}\right) \rightarrow\left(\mathcal{C}_{2}, T_{2}\right)$ be a monad opfunctor. Let $G: \mathcal{C}_{1} \rightarrow \mathcal{C}_{2}$, and let $i: G \rightarrow F$ be such that each component of $T_{2} i$ is monic. For each object $X$ of $\mathcal{C}_{1}$ let $\psi_{X}: G T_{1} X \rightarrow T_{2} G X$ be such that the following diagram commutes.

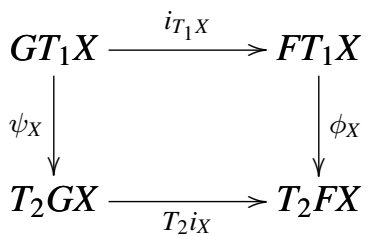

Then $(G, \psi)$ is a monad opfunctor and $i:(G, \psi) \rightarrow(F, \phi)$ is a transformation of monad opfunctors.

Proof A simple diagram chase shows that $\psi$ is natural, and then it is immediate that $i$ satisfies the conditions for a transformation of monad opfunctors. Next, we have

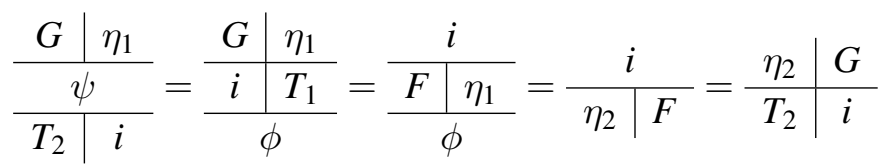

and hence $\begin{gathered}G \eta_{1} \\ \psi\end{gathered}=\eta_{2} G$. Next,

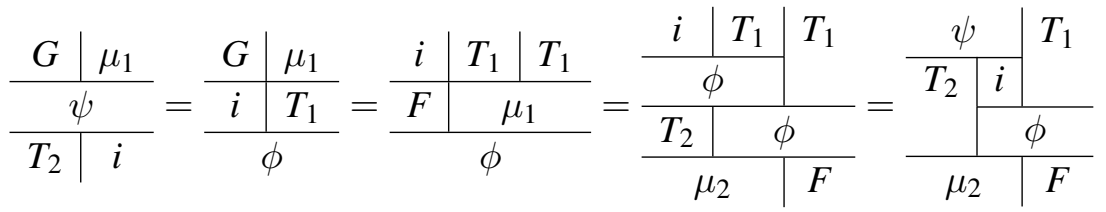

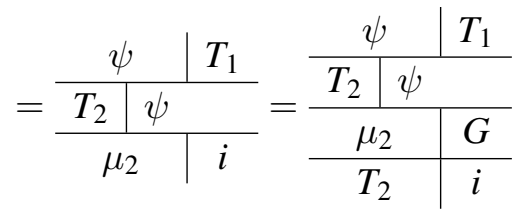


so

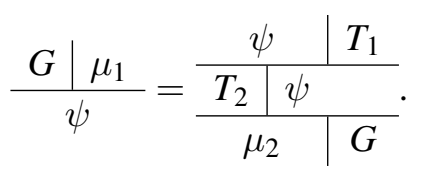

(Readers unfamiliar with this kind of vertical calculus can prove the result by diagram chasing.)

Lemma 2.8 Let $(F, \phi),(G, \psi),\left(G^{\prime}, \psi^{\prime}\right):\left(\mathcal{C}_{1}, T_{1}\right) \rightarrow\left(\mathcal{C}_{2}, T_{2}\right)$ be three monad opfunctors, and consider a diagram

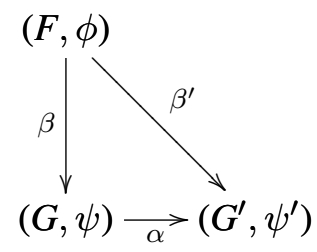

in which -

- $\beta^{\prime}$ and $\alpha$ are transformations of monad opfunctors,

- $\beta$ is a natural transformation of functors,

- the diagram commutes at the level of natural transformations of functors, and

- every component of $T_{2} \alpha$ is monic.

Then $\beta$ is a transformation of monad opfunctors.

Proof

$$
\frac{\frac{\beta \mid T_{1}}{\psi}}{T_{2} \mid \alpha}=\frac{\beta \mid T_{1}}{\alpha}=\frac{\beta^{\prime} \mid T_{1}}{\psi^{\prime}}=\frac{\phi}{\psi_{2} \mid \beta^{\prime}}=\frac{\phi}{T_{2}} \frac{\beta}{\alpha}
$$

and hence $\begin{gathered}\beta T_{1} \\ \psi\end{gathered}=\begin{gathered}\phi \\ T_{2} \beta\end{gathered}$.

\subsection{Continuous depos and their powerlocales}

When discussing the powerlocales, we shall find it useful to embed localic completions in (the localic form of) continuous dcpos (see, eg, [5]). For these, the powerlocales are well understood and constructed using finite powersets.

Journal of Logic \& Analysis 1:11 (2009) 
The usual definition is as follows. First, a poset $(P, \sqsubseteq)$ is directed complete (or a dcpo) if it has all directed joins. We write $\bigsqcup_{i}^{\uparrow} x_{i}$ for a directed join, the " $\uparrow$ " signifying that the family of $x_{i} \mathrm{~s}$ is indeed directed. If $x, y \in P$, we say $x$ is way below $y$, and write $x \ll y$, if whenever $y \sqsubseteq \bigsqcup_{i}^{\uparrow} z_{i}$ then $x \sqsubseteq z_{i}$ for some $i$. Then a dcpo $P$ is a continuous dcpo (or a domain) if for every $y \in P$ we have

$$
y=\bigsqcup^{\uparrow}(\ll y) .
$$

(Recall the notation from Section 1.2: $\ll y$ is $\{x \mid x \ll y\}$.) The default topology on a depo is the Scott topology, for which a subset $U$ is open if it is an upset and inaccessible by directed joins - ie if $\bigsqcup_{i}^{\uparrow} x_{i} \in U$ then $x_{i} \in U$ for some $i$.

We shall use the localic theory of continuous dcpos, with the characterization of [26] using continuous information systems.

Definition 2.9 A continuous information system (or cis or infosys) is a set $D$ equipped with an idempotent (transitive and interpolative) relation $\prec$.

A homomorphism between continuous information systems is a function that preserves $\prec$. We get a category cis of continuous information systems and homomorphisms.

An ideal of $(D, \prec)$ is a directed lower subset $I \subseteq D$ : in detail,

(1) $I$ is a lower set $(\prec I \subseteq I)$.

(2) I is inhabited (nullary directedness).

(3) If $s_{1}, s_{2} \in I$ then there is some $s \in I$ with $s_{1} \prec s$ and $s_{2} \prec s$ (binary directedness). (Consequently, $I$ is rounded, ie $I \subseteq \prec I$.)

We write $\operatorname{Idl}(D, \prec)$ (or often just $\operatorname{Idl}(D))$ for the locale whose points are the ideals of $(D, \prec)$. We can then present

$$
\begin{aligned}
\Omega \operatorname{Idl}(D, \prec) \cong \operatorname{Fr}\langle\uparrow s(s \in D)| \text { true } & \leq \bigvee_{s \in D} \uparrow s \\
& \uparrow s \wedge \uparrow t=\bigvee\{\uparrow u \mid s \prec u, t \prec u\}\rangle .
\end{aligned}
$$

We say a locale is a continuous depo if it is homeomorphic to one of the form $\operatorname{Idl}(D, \prec)$. (Note that this is not the same as saying that its frame is a continuous dcpo. In fact [26], a locale is a continuous dcpo iff its frame is constructively completely distributive - such frames are the Stone duals of continuous dcpos.) 
Proposition 2.10 Let $f:(D, \prec) \rightarrow(E, \prec)$ be a homomorphism of continuous information systems. Then we have a map $\operatorname{Idl}(f): \operatorname{Idl} D \rightarrow \operatorname{Idl} E$,

$$
\operatorname{Idl}(f)(I)=\prec f(I)
$$

where $f(I)$ denotes the direct image. In terms of opens, we have

$$
\operatorname{Idl}(f)^{*}(\uparrow t)=\bigvee\{\uparrow s \mid t \prec f(s)\}
$$

Proof It is straightforward to check that if $I$ is an ideal of $D$ then $\operatorname{Idl}(f)(I)$ as defined above is an ideal of $E$.

This gives a functor Idl : cis $\rightarrow$ Loc.

By [26], each powerlocale $P_{\sim} \operatorname{Idl}(D, \prec)$ is homeomorphic to $\operatorname{Idl}(\mathcal{F} D, \prec \sim)$ for a suitable idempotent order on the Kuratowski finite powerset $\mathcal{F} D$.

- The lower order has $S \prec_{L} T$ iff $\forall s \in S . \exists t \in T$. $s \prec t$.

- The upper order has $S \prec_{U} T$ iff $\forall t \in T$. $\exists s \in S$. $s \prec t$.

- The convex order has $S \prec_{C} T$ iff $S \prec_{L} T$ and $S \prec_{U} T$.

The notation $\prec_{L}, \prec_{U}$ and $\prec_{C}$ will be used uniformly by applying those subscripts to binary relations. Note that $\left(\prec_{U}\right)^{o p}=\left(\prec^{o p}\right)_{L}$ (or $\succ_{L}$ ). This gives a useful duality principle.

Proposition 2.11 We have three monads $\mathcal{F}_{\sim}$ on cis, given by

$$
\mathcal{F}_{\sim}(D, \prec)=(\mathcal{F} D, \prec \sim) .
$$

In each case, the unit and multiplication of the monad are the same as those for the $\mathcal{F}$ monad on Set (see Example 2.5).

Proof Checking is routine. One must check (i) $\mathcal{F}_{\sim}$ is functorial, and (ii) $\{-\}$ and $\bigcup$ are homomorphisms. Once these are done for $\mathcal{F}_{L}$, they follow for $\mathcal{F}_{U}$ by duality, and then for $\mathcal{F}_{C}$ by combining the two.

Definition 2.12 If $(D, \prec)$ is a continuous information system then we define a map $v_{\sim}^{\prime}: \operatorname{Idl} \mathcal{F}_{\sim}(D, \prec) \rightarrow P_{\sim} \operatorname{Idl}(D, \prec)$ by

$$
\begin{aligned}
v_{\sim}^{\prime *}(\diamond \uparrow s) & =\bigvee\left\{\uparrow T \mid\{s\} \prec_{L} T\right\}(\sim \text { stands for } \mathrm{L} \text { or } \mathrm{C}) \\
v_{\sim}^{\prime *}\left(\square \bigvee_{s \in S} \uparrow s\right) & =\bigvee\left\{\uparrow T \mid S \prec_{U} T\right\}(\sim \text { stands for } \mathrm{U} \text { or } \mathrm{C} ; S \in \mathcal{F} D) .
\end{aligned}
$$


Since the opens $\uparrow s$ form a base for $\operatorname{Idl}(D, \prec)$, and $\diamond$ preserves joins, it suffices to define $v_{\sim}^{\prime *}(\diamond U)$ for basic $U$. However, note that we also have

$$
\begin{aligned}
v_{\sim}^{\prime *}\left(\bigwedge_{s \in S} \diamond \uparrow s\right) & =\bigwedge_{s \in S} \bigvee\left\{\uparrow T \mid\{s\} \prec_{L} T\right\} \\
& =\bigvee\left\{\uparrow T \mid \text { for each } s \text { we have } T_{s} \text { with }\{s\} \prec_{L} T_{s} \prec \sim T\right\} \\
& =\bigvee\left\{\uparrow T \mid S \prec_{L} T\right\},
\end{aligned}
$$

thus giving a formal similarity with the $\square$ case. Since $\square$ preserves directed joins, it suffices to define $v_{\sim}^{\prime *}(\square U)$ for $U$ a finite join of basics.

[26, Theorem 4.3] shows that $v_{\sim}^{\prime}$ is a homeomorphism. The proof there is readily made topos-valid. (It uses cardinality of finite sets and decidable equality for their elements. However, this can easily be remedied by replacing the finite set with an enumeration, possibly with repetitions, of the elements.) The inverses for $v_{\sim}^{\prime *}$ are given by

$$
\uparrow S \mapsto \begin{cases}\bigwedge_{s \in S} \diamond \uparrow s & \text { (for } \mathrm{L} \text { ) } \\ \square\left(\bigvee_{s \in S} \uparrow s\right) & \text { (for } \mathrm{U} \text { ) } \\ \square\left(\bigvee_{s \in S} \uparrow s\right) \wedge \bigwedge_{s \in S} \diamond \uparrow s & \text { (for } \mathrm{C} \text { ) }\end{cases}
$$

Note that this proves that the class of continuous dcpos is closed under the powerlocales.

We show how this translates into actions on ideals.

Lemma 2.13 Let $(D, \prec)$ be a continuous information system, let $I$ be an ideal of $(\mathcal{F} D, \prec \sim)$, and let $S \in \mathcal{F} D$.

(1) ( is $L$ or $C$ ) $I$ is in the open $\bigwedge_{s \in S} \diamond \uparrow s$ for $P_{\sim} \operatorname{Idl}(D)$ iff $S \in \prec_{L} I$.

(2) ( $\sim$ is $U$ or $C$ ) $I$ is in the open $\square\left(\bigvee_{s \in S} \uparrow s\right.$ ) for $P \sim \operatorname{Idl}(D)$ iff $S \in \prec_{U} I$.

Note that if $\sim$ is $L$ or $U$ we have $\prec_{L} I$ and $\prec_{U} I$ (respectively) equal to $I$.

Proof The proof of [26, Theorem 4.3] deals explicitly with the Vietoris powerlocale where $\sim$ is C. From it we see that $\diamond \uparrow s$ corresponds to $\bigvee\{\uparrow T \mid T \cap(\succ s) \neq \emptyset\}$, which equals $\bigvee\left\{\uparrow T \mid\{s\} \prec_{L} T\right\}$. Now $I$ is in the open $\uparrow T$ iff $T \in I$, and so we can deduce that $I$ is in $\diamond \uparrow s$ iff $\{s\} \prec_{L} T$ for some $T \in I$. The first condition readily follows. Similarly, $\square\left(\bigvee_{s \in S} \uparrow s\right)$ corresponds to $\bigvee\{\uparrow T \mid T \subseteq \succ S\}$, which equals $\bigvee\left\{\uparrow T \mid S \prec_{U} T\right\}$.

[26] leaves to the reader the easier cases of the lower and upper powerlocales, but they yield the same conditions. 
Note the empty point for each powerlocale. For the lower, it is $I=\{\emptyset\}$ (every ideal contains $\emptyset$ ); for the upper it is $I=\mathcal{F} X$ (if an ideal contains $\emptyset$ then it contains every finite set); for the Vietoris it is $\{\emptyset\}$ (an ideal can contain either $\emptyset$ or non-empty sets, but not both). Excluding the empty set, we find that $v_{\sim}^{\prime}$ also gives a homeomorphism $\operatorname{Idl} \mathcal{F}_{\sim}^{+}(D, \prec) \cong P_{\sim}^{+} \operatorname{Idl}(D, \prec)$.

Proposition $2.14\left(\operatorname{Idl}, v_{\sim}^{\prime}\right):\left(\operatorname{cis}, \mathcal{F}_{\sim}\right) \rightarrow\left(\operatorname{Loc}, P_{\sim}\right)$ is a monad opfunctor.

Proof Using Proposition 2.10 and Lemma 2.13, it is easily checked on inverse image functions that $v_{\sim}^{\prime}$ is natural and that the conditions of Definition 2.6 are satisfied.

We shall also need the following.

Lemma 2.15 Let $(D, \prec)$ be a continuous information system.

(1) The map $\Downarrow: V \operatorname{Idl}(D) \rightarrow P_{L} \operatorname{Idl}(D)$ maps each ideal $I$ of $\left(\mathcal{F} D, \prec_{C}\right)$ to $\prec_{L} I$.

(2) The map $\Uparrow: V \operatorname{Idl}(D) \rightarrow P_{U} \operatorname{Idl}(D)$ maps each ideal $I$ of $\left(\mathcal{F} D, \prec_{C}\right)$ to $\prec_{U} I$.

Proof 1. Using Lemma 2.13 we have $S \in \Downarrow I$ iff $\Downarrow I$ is in $\bigwedge_{s \in S} \diamond \uparrow s$, ie iff $I$ is in $\bigwedge_{s \in S} \diamond \uparrow s$ since the inverse image function $\Omega \Downarrow$ takes each generator $\diamond U$ in the lower powerlocale to the one with the same name in the Vietoris. From Lemma 2.13 again this is equivalent to $S \in \prec_{L} I$.

2. This is similar.

\subsection{Generalized metric completion}

We now summarize the account of localic completion that appears in [33]. The description there is in terms of "spaces", on the understanding that classical mathematicians can interpret it in the conventional way, while locale theorists can read in a localic interpretation as described above. In the present paper our results are unavoidably localic, so we shall write of locales throughout.

$Q_{+}$is the set of positive rationals.

$\overleftarrow{[-\infty, \infty]}$ is the locale whose points are extended "upper reals", ie rounded upper sets of rationals. Classically, the finite upper reals (ie those for which the rounded upper set of rationals is inhabited but not the whole of $\mathbb{Q}$ ) are equivalent to ordinary reals, ie Dedekind sections. Constructively, however, there may be finite upper reals for 
which there is no corresponding lower set of rationals to make a Dedekind section. The arrow indicates the direction of the specialization order $\sqsubseteq$. This is the reverse of the numerical order $\leq(-\infty$ is top for $\sqsubseteq)$, because a large upper set of rationals denotes a numerically small upper real. We shall be particularly interested in $\overleftarrow{[0, \infty]}$, the locale of non-negative, extended upper reals, whose points are rounded upper sets of positive rationals.

The continuous arithmetic structure on $\overleftarrow{[0, \infty]}$ includes order, addition and multiplication, finitary max and min, and infinitary inf. It does not include any kind of subtraction (which would have to be contravariant in one argument, whereas continuous maps must preserve specialization order), or infinitary sup.

We now give the definition from [14], but using the locale $\overleftarrow{[0, \infty]}$ for the reals.

Definition 2.16 A generalized metric space (or gms) is a set $X$ equipped with a distance map $X(-,-): X^{2} \rightarrow \overleftarrow{[0, \infty]}$ satisfying

(zero self-distance)

$$
\begin{aligned}
& X(x, x)=0 \\
& X(x, z) \leq X(x, y)+X(y, z)
\end{aligned}
$$

(triangle inequality)

(By map we mean continuous map, between locales. We are treating the set $X^{2}$ as the corresponding discrete locale.)

If $X$ and $Y$ are two gms's, then a homomorphism from $X$ to $Y$ is a non-expansive function, ie a function $f: X \rightarrow Y$ such that $Y\left(f\left(x_{1}\right), f\left(x_{2}\right)\right) \leq X\left(x_{1}, x_{2}\right)$. We write gms for the category of generalized metric spaces and homomorphisms.

The opposite gms $X^{o p}$ has $X^{o p}(x, y)=X(y, x)$.

Remark 2.17 There is a predicate geometric theory whose models are the generalized metric spaces, and our notion of homomorphism is the natural one in that context.

Definition 2.18 If $X$ is a generalized metric space then we introduce the symbol " $B_{\delta}(x)$ ", a "formal open ball", as alternative notation for the pair $(x, \delta) \in X \times Q_{+}$. We write

$$
B_{\varepsilon}(y) \subset B_{\delta}(x) \text { if } X(x, y)+\varepsilon<\delta
$$

(in other words, if $\varepsilon<\delta$ and $X(x, y)<\delta-\varepsilon$ ) and say in that case that $B_{\varepsilon}(y)$ refines $B_{\delta}(x)$.

Definition 2.19 Let $X$ be a generalized metric space. A subset $F$ of $X \times Q_{+}$is a Cauchy filter if - 
(1) $F$ is upper with respect to $\subset$, ie if $B_{\varepsilon}(y) \subset B_{\delta}(x)$ and $B_{\varepsilon}(y) \in F$ then $B_{\delta}(x) \in F$.

(2) If $B_{\delta}(x) \in F$ and $B_{\delta^{\prime}}\left(x^{\prime}\right) \in F$ then there is some $B_{\varepsilon}(y) \in F$ with $B_{\varepsilon}(y) \subset B_{\delta}(x)$ and $B_{\varepsilon}(y) \subset B_{\delta^{\prime}}\left(x^{\prime}\right)$.

(3) For every $\delta \in Q_{+}$there is some $x \in X$ such that $B_{\delta}(x) \in F$.

Definition 2.20 [33] Let $X$ be a generalized metric space. Then its localic completion $\bar{X}$ (or $\operatorname{Comp}(X))$ is the locale whose points are the Cauchy filters of formal open balls. If $f: X \rightarrow Y$ is a gms homomorphism then $\operatorname{Comp}(f)=\bar{f}: \bar{X} \rightarrow \bar{Y}$ is defined by

$$
\bar{f}(F)=\supset\left\{B_{\delta}(f(x)) \mid B_{\delta}(x) \in F\right\} .
$$

These together give a functor Comp: gms $\rightarrow$ Loc.

Proposition 2.21 Let $f, g: X \rightarrow Y$ be homomorphisms of gms's such that for all $x \in X, Y(f(x), g(x))=0$. Then $\bar{f} \sqsubseteq \bar{g}$.

Proof Suppose $F$ is a Cauchy filter for $X$ and $B_{\varepsilon}(y) \in \bar{f}(F)$ with $B_{\varepsilon}(y) \supset B_{\delta}(f(x))$, $B_{\delta}(x) \in F$. Then

$$
Y(y, g(x))+\delta \leq Y(y, f(x))+Y(f(x), g(x))+\delta=Y(y, f(x))+\delta<\varepsilon
$$

so $B_{\varepsilon}(y) \supset B_{\delta}(g(x))$ and $B_{\varepsilon}(y) \in \bar{g}(F)$.

Definition 2.22 Let $X$ be a generalized metric space. A subset $F$ of $X \times Q_{+}$is a left $X$-module if it is a rounded upper set of formal open balls -

(1) $F$ is upper with respect to $\subset$ (as in Definition 2.19).

(2) $F$ is rounded, ie if $B_{\delta}(x) \in F$ then there is some $B_{\varepsilon}(y) \in F$ with $B_{\varepsilon}(y) \subset B_{\delta}(x)$.

We write $X$-Mod for the locale whose points are the left $X$-modules.

(The term "module" is explained in [33] in terms of Lawvere's use of enriched categories. In the present paper those ideas are less relevant but we keep the term.)

\section{Powerspaces}

If $X$ is a gms, we shall define three powerspaces $\mathcal{F}_{\sim X}$ on it, where $\sim$ can stand for $L$ (lower), $U$ (upper) or $C$ (convex, or the Vietoris powerspace). They are defined by 
three generalized metrics on the finite powerset $\mathcal{F} X$,

$$
\begin{aligned}
& \mathcal{F}_{L} X(S, T)=\max _{x \in S} \min _{y \in T} X(x, y) \\
& \mathcal{F}_{U} X(S, T)=\max _{y \in T} \min _{x \in S} X(x, y) \\
& \mathcal{F}_{C} X(S, T)=\max \left(\mathcal{F}_{L} X(S, T), \mathcal{F}_{U} X(S, T)\right) .
\end{aligned}
$$

The finite sets $S$ and $T$ may be empty. We have $\mathcal{F}_{L} X(\emptyset, T)=0$ for all $T$, and $\mathcal{F}_{L} X(S, \emptyset)=\infty$ if $S$ is non-empty. $\mathcal{F}_{U} X$ is similar but the other way round, and $\mathcal{F}_{C} X(S, T)=\infty$ if just one of $S, T$ is empty, but 0 if both are (fortunately for the zero self-distance law).

Clearly $\mathcal{F}_{C} X$ is the Hausdorff metric restricted to finite sets - hence it is calculated in a finitary way, without recourse to limits. The other two are less familiar but clearly derive from separating out parts of the Hausdorff metric.

A useful duality principle is that $\mathcal{F}_{U} X=\left(\mathcal{F}_{L}\left(X^{o p}\right)\right)^{o p}$.

If $X$ is symmetric then so is $\mathcal{F}_{C} X$, but the other two are not.

We write $\mathcal{F}_{\sim}^{+} X$ for the three positive powerspaces, ie restricted to non-empty finite subsets.

Proposition 3.1 The monad structure of $\mathcal{F}$ extends to all three powerspaces, giving monads on gms.

Proof We need to check that the functions involved are non-expansive. First, the unit $\{-\}: X \rightarrow \mathcal{F} X$ is obviously an isometry for all three.

For the rest, we first consider $\mathcal{F}_{L}$. The multiplication $\bigcup: \mathcal{F}^{2} X \rightarrow \mathcal{F} X, \mathcal{U} \mapsto \bigcup \mathcal{U}$, is a homomorphism (non-expansive) because

$$
\begin{aligned}
\mathcal{F}_{L}^{2} X(\mathcal{U}, \mathcal{V})<q & \Longleftrightarrow \forall U \in \mathcal{U} . \exists V \in \mathcal{V} . \mathcal{F}_{L} X(U, V)<q \\
& \Longleftrightarrow \forall U \in \mathcal{U} . \exists V \in \mathcal{V} . \forall u \in U . \exists v \in V . X(u, v)<q \\
& \Longleftrightarrow \forall U \in \mathcal{U} . \forall u \in U . \exists V \in \mathcal{V} . \exists v \in V . X(u, v)<q \\
& \Longleftrightarrow \forall u \in \bigcup \mathcal{U} . \exists v \in \bigcup \mathcal{V} . X(u, v)<q \\
& \Longleftrightarrow \mathcal{F}_{L} X(\bigcup \mathcal{U}, \bigcup \mathcal{V})<q .
\end{aligned}
$$


Now if $f: X \rightarrow Y$ is a homomorphism then so is $\mathcal{F} f$ (where $\mathcal{F} f(S)$ is the direct image $f(S))$ because

$$
\begin{aligned}
\mathcal{F}_{L} X(S, T)<q & \Longleftrightarrow \forall x \in S . \exists y \in T . X(x, y)<q \\
& \Longleftrightarrow \forall x \in S . \exists y \in T . Y(f(x), f(y))<q \\
& \Longleftrightarrow \forall x^{\prime} \in \mathcal{F} f(S) . \exists y^{\prime} \in \mathcal{F} f(T) . Y\left(x^{\prime}, y^{\prime}\right)<q \\
& \Longleftrightarrow \mathcal{F}_{L} Y(\mathcal{F} f(S), \mathcal{F} f(T))<q
\end{aligned}
$$

The corresponding results for the upper metric follow by duality, and then those for the Vietoris metric are immediate.

\section{The ball domain}

A technique pioneered in [3] (working classically) is to prove results about complete metric spaces by embedding them in continuous dcpos, the ball domains, so that limits in the metric space can be found using directed joins in the dcpo. A simple example then is that the Banach fixed point theorem follows easily from the dcpo fixed point theorems. They also deal with compact subspaces by using powerdomain techniques on the ball domain. Our working will depend on an analogous construction, the principal benefit being to allow us to exploit the way powerlocales are constructed for continuous dcpos. Ball domains for gms's have also been described in [20], and we shall compare our construction with the work there.

If $X$ is a metric space, then Edalat and Heckmann [3] define the ball domain $\mathbf{B}(X)$ to be the product $X \times[0, \infty)$, ordered by the non-strict analogy of our $\supset$ on $X \times Q_{+}$. They call the elements of $\mathbf{B}(X)$ formal balls on $X$. Although $\mathbf{B}(X)$ is not a dcpo in general, they define a Scott topology on it and show (theorem 13) that $X$ embeds in it as the maximal elements $(x, 0)$. Moreover, they define the way below relation and show (section 2.6) that the metric completion $\bar{X}$ embeds in the ideal completion of $\mathbf{B}(X)$ with respect to way below.

Clearly their formal balls are more general than ours in allowing radii that are 0 or irrational. Our ball domain will go further than theirs by allowing centres to be in the completed space. In other words, in a single step we shall complete on both radii and centres.

Note the elementary fact that $\subset$ and $\supset$ are idempotent relations on $X \times Q_{+}$. We shall write ball $(X)$ for the continuous information system $\left(X \times Q_{+}, \supset\right)$. This extends to a functor ball: gms $\rightarrow$ cis, by ball $(f)\left(B_{\delta}(x)\right)=B_{\delta}(f(x))$. 
Definition 4.1 The ball domain functor Ball: $\mathbf{g m s} \rightarrow$ Loc is Idl $\circ$ ball .

In practice it is more natural to view the points of the ball domain as filters of $X \times Q_{+}$ with respect to $\subset$, rather than ideals with respect to $\supset$.

Proposition 4.2 A point of $\operatorname{Ball}(X)$ is a subset $G$ of $X \times Q_{+}$satisfying the conditions (1) and (2) of Definition 2.19, and in addition that for some $\delta$ there is $x$ with $B_{\delta}(x) \in G$.

Proof Condition (1) says that $G$ is rounded lower with respect to $\supset,(2)$ is binary directedness and the new condition is nullary directedness, ie inhabitedness.

Hence $\bar{X}$ embeds as a sublocale of the continuous depo $\operatorname{Ball}(X)$, and in turn $\operatorname{Ball}(X)$ embeds as a sublocale of $X$-Mod (Definition 2.22). The points of $X$-Mod are the rounded upper sets of balls, those of $\operatorname{Ball}(X)$ are the filters, and those of $\bar{X}$ are the Cauchy filters.

Definition 4.3 We write $i:$ Comp $\rightarrow$ Ball for the natural transformation whose component at a gms $X$ is the embedding $\bar{X} \hookrightarrow \operatorname{Ball}(X)$.

Definition 4.4 For any gms $X$, we define $\phi: \mathcal{F} X \times Q_{+} \rightarrow \mathcal{F}\left(X \times Q_{+}\right)$by $\phi\left(B_{\varepsilon}(S)\right)=$ $\left\{B_{\varepsilon}(x) \mid x \in S\right\}$.

Lemma 4.5 If $B_{\delta}(S) \supset B_{\varepsilon}(T)$ (with respect to the metric on $\mathcal{F}_{\sim} X$, where $\sim$ is $L, U$ or $C)$, then $\phi\left(B_{\delta}(S)\right) \supset \sim \phi\left(B_{\varepsilon}(T)\right)$. If we are given that $\varepsilon<\delta$ then the converse also holds.

Proof We have $\mathcal{F}_{\sim} X(S, T)+\varepsilon<\delta$. First, when $\sim$ is $\mathrm{L}$ the condition is equivalent to $\varepsilon<\delta$ and

$$
\forall x \in S . \exists y \in T . B_{\delta}(x) \supset B_{\varepsilon}(y),
$$

and these together imply $\phi\left(B_{\delta}(S)\right) \supset_{L} \phi\left(B_{\varepsilon}(T)\right)$ - in fact, if we are given that $\varepsilon<\delta$ then the reverse implication also holds. (If $S$ is non-empty, then $\phi\left(B_{\delta}(S)\right) \supset_{L} \phi\left(B_{\varepsilon}(T)\right.$ ) already implies $\varepsilon<\delta$. However, for the lower order, we have $\phi\left(B_{\delta}(\emptyset)\right) \supset_{L} \phi\left(B_{\varepsilon}(T)\right)$ for all $\delta, \varepsilon, T$.) The case when $\sim$ is $\mathrm{U}$ can be proved either similarly or by duality, and then the case when $\sim$ is $\mathrm{C}$ follows.

Proposition 4.6 (ball, $\left.\phi_{\sim}\right)$ is a monad opfunctor from $\left(\mathrm{gms}, \mathcal{F}_{\sim}\right)$ to $\left(\operatorname{cis}, \mathcal{F}_{\sim}\right)$. 
Proof Lemma 4.5 shows that each component of $\phi_{\sim}$ is a homomorphism of continuous information systems, and then naturality is easily checked. The rest is routine checking.

Definition 4.7 The monad opfunctor $\left(\right.$ Ball, $\left.\phi_{\sim}^{\prime}\right):\left(\mathbf{g m s}, \mathcal{F}_{\sim}\right) \rightarrow\left(\right.$ Loc,$\left.P_{\sim}\right)$ is defined as the composite of (ball, $\left.\phi_{\sim}\right)$ and $\left(\operatorname{Idl}, v_{\sim}^{\prime}\right)$.

Thus $\phi_{\sim}^{\prime}=\underset{v_{\sim}^{\prime} \text { ball }}{\operatorname{Idl} \phi_{\sim}}: \operatorname{Ball}\left(\mathcal{F}_{\sim} X\right) \rightarrow P_{\sim} \operatorname{Ball}(X)$, so $\phi_{\sim}^{\prime}(X)(I)=(\supset \sim \phi(I))$, where $\phi(I)$ is the direct image.

We finish this section by showing how our ball domain relates classically to those of [3] and [20]. The projection $X \times Q_{+} \rightarrow Q_{+}$is a cis-homomorphism, and so lifts to a radius map $r: \operatorname{Ball}(X) \rightarrow \overleftarrow{[0, \infty)}=\operatorname{Idl}\left(Q_{+},>\right)$by $r(G)=\inf \left\{q \mid B_{q}(x) \in G\right\}$, an upper real. Lifting the cis-homomorphism $\left(X \times Q_{+}\right) \times Q_{+} \rightarrow X \times Q_{+},\left(B_{\delta}(x), q\right) \mapsto B_{\delta+q}(x)$, we also obtain a map + : $\operatorname{Ball}(X) \times \overleftarrow{[0, \infty)} \rightarrow \operatorname{Ball}(X)$, defined by

$$
G+a=\left\{B_{\delta+q}(x) \mid B_{\delta}(x) \in G, a<q\right\} .
$$

Note that $r(G+a)=r(G)+a$, and $G$ is Cauchy iff $r(G)=0$.

Proposition 4.8 (Classically) Let $X$ be a gms. Then the map $+: \bar{X} \times \overleftarrow{[0, \infty)} \rightarrow$ $\operatorname{Ball}(X)$ is a bijection on points.

Proof Let $G$ be a filter in $X \times Q_{+}$. We define its centre $c(G)$ to be $\left\{B_{\delta}(x) \mid \exists B_{q}(x) \in\right.$ $G . q-\delta<r(G)\}$. (Note - constructively, the upper real $r(G)$ is approximated from above. It is therefore constructively illegitimate to use approximations from below, as in $q-\delta<r(G)$.)

If $F$ is a Cauchy filter and $a$ is a non-negative upper real, then $c(F+a)=F$, ie

$$
B_{\delta}(x) \in F \Longleftrightarrow \exists \delta^{\prime}, q .\left(B_{\delta^{\prime}}(x) \in F \text { and } \delta^{\prime}+q-\delta<a<q\right) .
$$

The $\Leftarrow$ direction follows because $\delta^{\prime}<\delta$. For $\Rightarrow$, we can find $\delta^{\prime}<\delta$ such that $B_{\delta^{\prime}}(x) \in F$, and then $q$ such that $q-\left(\delta-\delta^{\prime}\right)<a<q$.

Now suppose $G$ is a filter in $X \times Q_{+}$. We must show $G=c(G)+r(G)$, which works out as equivalent to

$$
B_{q}(x) \in G \Longleftrightarrow \exists \delta, q^{\prime} .\left(B_{q^{\prime}}(x) \in G \text { and } q^{\prime}-\delta<r(G)<q-\delta\right) .
$$

$\Leftarrow$ is obvious. For $\Rightarrow$, find $q^{\prime}<q$ such that $B_{q^{\prime}}(x) \in G$ and then $r$ such that $r-\left(q-q^{\prime}\right)<r(G)<r<q$, and then put $\delta=q-r$. 
Of course, the map + is not a homeomorphism. As can easily be seen from the case of symmetric $X$, the specialization orders on $\bar{X} \times \overleftarrow{[0, \infty)}$ and $\operatorname{Ball}(X)$ are different. The classical result shows that for symmetric $X$, the points of our $\operatorname{Ball}(X)$ are the same as those of the ball domain $\mathbf{B}(\bar{X})$ in [3].

We should also compare our formal balls with those of [20], which deals with the gms case (albeit classically). Rutten defines the formal ball $F\langle r, x\rangle(r \in[0, \infty], x \in X)$ to be a function from $X$ to $[0, \infty]$, given by $y \mapsto r+X(x, y)$. This is in fact (to use the terminology of [33]) a right module over $X$, in other words a point of $X^{o p}-$ Mod. When we consider Rutten's $F\langle r, x\rangle$ with respect to $X^{o p}$ we find that it is just our $\mathcal{Y}(x)+r$, where $\mathcal{Y}: X \rightarrow \bar{X}$ is the Yoneda embedding, $\mathcal{Y}(x)=\left\{B_{\varepsilon}(y) \mid X(y, x)<\varepsilon\right\}$. Note that Rutten allows $r=\infty$ here, which does not give a point of $\operatorname{Ball}(X)$ - as a subset of $X \times Q_{+}$it is empty. Rutten's ball domain $\mathcal{F}$ over $X$ is defined to be the set of right modules $F\langle r, x\rangle$, so his ball domain over $X^{o p}$ (apart from the infinite balls) is included in the set of points of $\operatorname{Ball}(X)$. Once again, our ball domain has the extra generality of allowing the centre to be in the completion of $X$.

\section{Main results}

Our strategy now is to show that we have a factorization

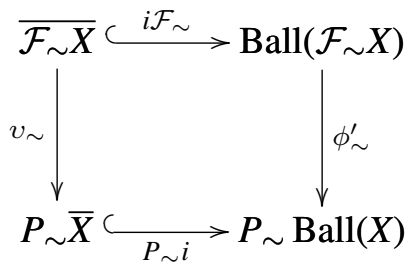

with $v_{\sim}$ in fact a homeomorphism (though $\phi_{\sim}^{\prime}$ is not), giving a monad opfunctor $\left(\right.$ Comp, $\left.v_{\sim}\right):\left(\mathbf{g m s}, \mathcal{F}_{\sim}\right) \rightarrow\left(\mathbf{L o c}, P_{\sim}\right)$ such that $i:\left(\right.$ Comp, $\left.v_{\sim}\right) \rightarrow\left(\right.$ Ball, $\left.\phi_{\sim}^{\prime}\right)$ is a transformation of monad opfunctors.

There are two main steps in the proof. The first (Section 5.1) is to identify those points of $P \sim \operatorname{Ball}(X)$ that are in the sublocale $P \sim \bar{X}$, and the second (Section 5.2) is to describe the factorization through a homeomorphism. The process will also describe how points of $\overline{\mathcal{F}_{\sim X}}$ (Cauchy filters of balls for $\mathcal{F}_{\sim X}$ ) satisfy the $\square$ and $\diamond$ opens of $P \sim \operatorname{Ball}(X)$. This will be needed in order to see how they correspond with sublocales of $\bar{X}$. 


\subsection{The embeddings $P_{\sim} \bar{X} \hookrightarrow P_{\sim} \operatorname{Ball}(X)$}

[3, theorem 22] use their domain theoretic results to deal not only with points of a complete metric space $X$, but also with compact subspaces. For this they use the Plotkin powerdomain (of which the Vietoris powerlocale is the localic analogue) of their ball domain. They show (their theorem 22) that the compact subspaces of $X$ are in bijection with the rounded ideals $I$ of finite non-empty subsets of $X \times[0, \infty)$ for which

$$
\inf _{S \in I} \max _{(x, r) \in S} r=0
$$

We now give constructive localic analogues of their result, for all three powerlocales and covering the generalized metric case. We shall replace $X \times[0, \infty)$ by $X \times Q_{+}$(and drop any assumption that $X$ is complete), and then the results surveyed in Section 2.3 show that the powerlocales of $\operatorname{Ball}(X)$ are got as the ideal completions of $\mathcal{F}\left(X \times Q_{+}\right)$ under suitable orders $\supset_{\sim}$. Since $\bar{X}$ embeds in $\operatorname{Ball}(X)$, it follows that $P_{\sim}(\bar{X})$ embeds in $\operatorname{Idl}\left(\mathcal{F}\left(X \times Q_{+}\right), \supset \sim\right)$. Our task in this section is to identify which ideals of $\mathcal{F}\left(X \times Q_{+}\right)$ are in the sublocale $P_{\sim}(\bar{X})$.

[2] also examine powerdomains for generalized metric spaces. Their most detailed working is for the lower. They work in the case where $X$ is complete but has a "basis" $B$, and the lower powerdomain is then a certain subset of $\widehat{B}=B-$ Mod. In fact (our Proposition 5.1), $\widehat{B}$ can be understood as $P_{L} \operatorname{Ball}(X)$, so we share a basic approach. However, their characterization of that subset is very different from ours, being described in terms of limits of sequences. They also show (their corollary 7.11) that this subset is isomorphic to the completion of the finite powerset of $B$ with the lower metric. It is difficult to compare this account directly with ours, since they are throughout using not only classical, spatial reasoning, but also sequencewise definitions of completeness and completion ([33] shows that netwise definitions are needed to get a good classical correspondence). They also (their section 7.4) sketch the definition of upper and convex powerdomains, and state without proof that they can be obtained by completing the finite powerset with upper or convex metric.

Before proving the main results of this section, let us give the following result that relates them to the approach of [2].

Proposition 5.1 Let $X$ be a gms. Then $X-M o d$ is homeomorphic to $P_{L}(\operatorname{Ball}(X))$.

Proof It is straightforward to show that, for any continuous information system $D$, the rounded downsets of $D$ are equivalent to ideals of $\mathcal{F}_{L} D$, hence to points of $P_{L}(\operatorname{Idl}(D))$ : 
a rounded downset $I$ corresponds to the ideal $J=\{S \mid S \subseteq I\}$, and, inversely, $J$ corresponds to $I=\{s \mid\{s\} \in J\} .^{2}$

Now the points of $X$-Mod, the rounded upsets of formal open balls, are the rounded downsets with respect to the refinement order $\supset$ used in ball $(X)$, and hence ideals of $\mathcal{F}_{L}$ ball $(X)$. We conclude that $X-\operatorname{Mod}$ is homeomorphic to $P_{L}(\operatorname{Ball}(X))$.

Note that reversing the order on the information system $D$ corresponds to taking the Lawson dual ${ }^{3}$ of the continuous dcpo, its points being the Scott open filters of points of $\operatorname{Idl}(D)$.

We now prove the main results of this section. In each $P_{\sim} \operatorname{Ball}(X) \cong \operatorname{Idl}(\mathcal{F}(X \times$ $\left.\left.Q_{+}\right), \supset_{\sim}\right)$, we identify which points correspond to those in the image of $P_{\sim} \bar{X}$. Following [3], if $S \in \mathcal{F}\left(X \times Q_{+}\right)$then we write $r S=\max \left\{\delta \mid B_{\delta}(x) \in S\right\} \in \mathbb{Q}$, and if $J \subseteq \mathcal{F}\left(X \times Q_{+}\right)$then we write $\bar{r} J=\inf \{r S \mid S \in J\} \in[0, \infty]$. In the context of [3] (with $X$ complete and symmetric) they prove that non-empty compact subspaces of $X$ correspond to ideals $J$ of $\left(\mathcal{F}^{+}(X \times[0, \infty)), \supset_{C}\right)$ for which $\bar{r} J=0$.

We shall strengthen the condition $\overline{\mathrm{r}} J=0$, to one saying that for every $\varepsilon \in Q_{+}$, every $S \in J$ has a refinement $T \in J$ with $r T<\varepsilon$. For the upper and convex cases (which deal with compact subspaces) this is equivalent to $\bar{r} J=0$, because if $S \supset_{U} T$ then $r S>r T$. However, in the lower case our condition is stronger.

In proving the theorem, we shall consider the locale embedding $\bar{X} \rightarrow \operatorname{Ball}(X)$ in terms of frame presentations. From the definition of the points as filters of balls, we see immediately that the frame for $\operatorname{Ball}(X)$ can be presented as

$$
\begin{gathered}
\Omega \operatorname{Ball}(X)=\operatorname{Fr}\left\langle B_{\delta}(x)\left(x \in X, \delta \in Q_{+}\right)\right| \\
B_{\delta}(x) \wedge B_{\delta^{\prime}}\left(x^{\prime}\right)=\bigvee\left\{B_{\varepsilon}(y) \mid B_{\varepsilon}(y) \subset B_{\delta}(x) \text { and } B_{\varepsilon}(y) \subset B_{\delta^{\prime}}\left(x^{\prime}\right)\right\} \\
\left(x, x^{\prime} \in X, \delta, \delta^{\prime} \in Q_{+}\right) \\
\text {true } \left.=\bigvee\left\{B_{\delta}(x) \mid x \in X, \delta \in Q_{+}\right\}\right\rangle
\end{gathered}
$$

while the sublocale $\bar{X}$ has the extra relations

$$
\text { true }=\bigvee_{x \in X} B_{\varepsilon}(x)\left(\varepsilon \in Q_{+}\right)
$$

corresponding to the Cauchy property.

\footnotetext{
${ }^{2}$ The equivalence here is essentially that remarked on in [26, after theorem 4.3], using the fact that the rounded downsets of $D$ are the rounded upsets of $D^{o p}$ and hence the opens of $\operatorname{Idl}\left(D^{o p}\right)$. Thus $\Omega \operatorname{Idl} D$ is presented, as continuous dcpo, by the information system $\mathcal{F}_{L} D^{o p} \cong\left(\mathcal{F}_{U} D\right)^{o p}$.

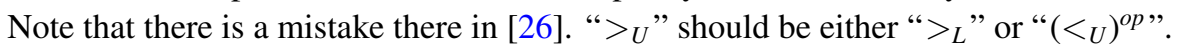

${ }^{3}$ This is sometimes known as the Hoffmann-Lawson dual, since - according to [7] - it was discovered independently by Hoffmann and by Lawson.
} 
Theorem 5.2 Let $J$ be a point of $\operatorname{Idl}\left(\mathcal{F}\left(X \times Q_{+}\right), \supset_{\sim}\right) \cong P_{\sim} \operatorname{Ball}(X)$. Then $J$ is in the image of $P \sim \bar{X}$ iff for all $S \in J$ and $\varepsilon \in Q_{+}$, there is some $T \in J$ with $S \supset \sim T$ and $r T<\varepsilon$.

Proof $\sim$ is L: Let $Y \hookrightarrow Z$ be an arbitrary locale embedding, with $\Omega Y$ presented over $\Omega Z$ by relations $a \leq b$ for $(a, b) \in R \subseteq \Omega Z \times \Omega Z$. By a routine application of the coverage theorem (see [28]), we have

$$
\Omega Y \cong \operatorname{SupLat}\langle\Omega Z \text { (qua SupLat) } \mid a \wedge c \leq b \wedge c((a, b) \in R, c \in \Omega Z)\rangle
$$

and it follows that

$$
\begin{aligned}
\Omega P_{L} Y & =\operatorname{Fr}\langle\Omega Y \text { (qua SupLat) }\rangle \\
& \cong \operatorname{Fr}\langle\Omega Z \text { (qua SupLat) } \mid a \wedge c \leq b \wedge c((a, b) \in R, c \in \Omega Z)\rangle \\
& \cong \operatorname{Fr}\left\langle\Omega P_{L} Z \text { (qua Fr) } \mid \diamond(a \wedge c) \leq \diamond(b \wedge c)((a, b) \in R, c \in \Omega Z)\right\rangle .
\end{aligned}
$$

Clearly it suffices to take the opens $c$ just from a base of $Z$.

In our present case we have $Y=\bar{X}, Z=\operatorname{Ball}(X)$, with $\Omega \bar{X}$ presented over $\Omega \operatorname{Ball}(X)$ by relations true $\leq \bigvee_{y \in X} B_{\varepsilon}(y)(\varepsilon>0)$. Hence, using the fact that the $B_{\delta}(x)$ 's are a base for $\operatorname{Ball}(X)$, we find that $\Omega P_{L} \bar{X}$ is presented over $\Omega P_{L} \operatorname{Ball}(X)$ by relations

$$
\begin{aligned}
\diamond B_{\delta}(x) & \leq \bigvee_{y \in X} \diamond\left(B_{\delta}(x) \wedge B_{\varepsilon}(y)\right) \\
& =\bigvee\left\{\diamond B_{\varepsilon^{\prime}}\left(y^{\prime}\right) \mid B_{\delta}(x) \supset B_{\varepsilon^{\prime}}\left(y^{\prime}\right) \text { and } \varepsilon^{\prime}<\varepsilon\right\}
\end{aligned}
$$

We now apply Lemma 2.13 (1). Suppose $J$ satisfies the relations, and suppose $S \in J$ and $\varepsilon \in Q_{+}$. For each $B_{\delta}(x) \in S$ we have $\left\{B_{\delta}(x)\right\} \in J$ and so there is some $\left\{B_{\varepsilon^{\prime}}\left(y^{\prime}\right)\right\} \in J$ with $B_{\varepsilon^{\prime}}\left(y^{\prime}\right) \subset B_{\delta}(x)$ and $\varepsilon^{\prime}<\varepsilon$. Taking those balls $B_{\varepsilon^{\prime}}\left(y^{\prime}\right)$ together, we can find a finite set $T \in J$ such that $S \supset_{C} T$ - hence $S \supset_{L} T-$ and $\mathrm{r} T<\varepsilon$. The converse is obvious.

$\sim$ is $\mathrm{U}$ : This time for the arbitrary locale embedding $Y \hookrightarrow Z$, by a routine application of the preframe coverage theorem [12], we have

$$
\Omega Y \cong \operatorname{PreFr}\langle\Omega Z \text { (qua PreFr) } \mid a \vee c \leq b \vee c((a, b) \in R, c \in \Omega Z)\rangle .
$$

It follows that

$$
\begin{aligned}
\Omega P_{U} Y & =\operatorname{Fr}\langle\Omega Y \text { (qua PreFr) }\rangle \\
& \cong \operatorname{Fr}\langle\Omega Z \text { (qua PreFr) } \mid a \vee c \leq b \vee c((a, b) \in R, c \in \Omega Z)\rangle \\
& \cong \operatorname{Fr}\left\langle\Omega P_{U} Z \text { (qua Fr ) } \mid \square(a \vee c) \leq \square(b \vee c)((a, b) \in R, c \in \Omega Z)\right\rangle
\end{aligned}
$$


In our present case with relations true $\leq \bigvee_{y} B_{\varepsilon}(y)(\varepsilon>0)$, the opens $c$ appearing above make no difference (true $\vee c=$ true). Hence we find that $\Omega P_{U} \bar{X}$ is presented over $\Omega P_{U} \operatorname{Ball}(X)$ by relations

$$
\text { true } \leq \square \bigvee_{y \in X} B_{\varepsilon}(y)=\bigvee^{\uparrow}\left\{\square \bigvee_{y \in T^{\prime}} B_{\varepsilon}(y) \mid T^{\prime} \in \mathcal{F} X\right\}
$$

We now apply Lemma 2.13 (2). Suppose $J$ satisfies the relations, and suppose $S \in J$ and $\varepsilon \in Q_{+}$. From the relations we can find $T^{\prime} \in \mathcal{F} X$ such that $\left\{B_{\varepsilon}(y) \mid y \in T^{\prime}\right\} \in J$. Let $T \in J$ be such that $S \supset_{U} T$ and $\left\{B_{\varepsilon}(y) \mid y \in T^{\prime}\right\} \supset_{U} T$. Then $\mathrm{r} T<\varepsilon$ and $T$ is as required. Conversely, suppose the condition holds. Let $\varepsilon \in Q_{+}$. We can find $S \in J$ (which is inhabited), and so there is some $T \in J$ with $S \supset_{U} T$ and $\mathrm{r} T<\varepsilon$. If $T^{\prime}=\left\{y \mid \exists \alpha . B_{\alpha}(y) \in T\right\}$ then $\left\{B_{\varepsilon}(y) \mid y \in T^{\prime}\right\} \in J$ as required.

$\sim$ is $\mathrm{C}$ : This time for an arbitrary locale embedding $Y \hookrightarrow Z$, combining the calculations for the lower and upper cases, we get

$$
\begin{gathered}
\Omega V Y=\operatorname{Fr}\langle\Omega Y \text { (qua SupLat), } \Omega Y(\text { qua PreFr })| \\
\square d \wedge \diamond e \leq \diamond(d \wedge e), \\
\square(d \vee e) \leq \square d \vee \diamond e\rangle \\
\cong \operatorname{Fr}\langle\Omega V Z \text { (qua Fr) }| \diamond(a \wedge c) \leq \diamond(b \wedge c), \square(a \vee c) \leq \square(b \vee c) \\
((a, b) \in R, c \in \Omega Z)\rangle .
\end{gathered}
$$

In our present case, as before, these relations reduce to

$$
\begin{aligned}
\diamond B_{\delta}(x) & \leq \bigvee\left\{\diamond B_{\varepsilon^{\prime}}\left(y^{\prime}\right) \mid B_{\delta}(x) \supset B_{\varepsilon^{\prime}}\left(y^{\prime}\right) \text { and } \varepsilon^{\prime}<\varepsilon\right\} \\
\text { true } & \leq \bigvee^{\uparrow}\left\{\square \bigvee_{y \in T^{\prime}} B_{\varepsilon}(y) \mid T^{\prime} \in \mathcal{F} X\right\} .
\end{aligned}
$$

However, given the second, we have the first (in fact they are equivalent): for

$$
\begin{aligned}
\diamond B_{\delta}(x) & \leq \bigvee^{\uparrow}\left\{\diamond B_{\delta}(x) \wedge \square \bigvee_{y \in T^{\prime}} B_{\varepsilon}(y) \mid T^{\prime} \in \mathcal{F} X\right\} \\
& \leq \bigvee^{\uparrow}\left\{\diamond\left(B_{\delta}(x) \wedge \bigvee_{y \in T^{\prime}} B_{\varepsilon}(y)\right) \mid T^{\prime} \in \mathcal{F} X\right\} \\
& =\bigvee_{y \in X} \diamond\left(B_{\delta}(x) \wedge B_{\varepsilon}(y)\right) \leq \text { RHS of first. }
\end{aligned}
$$

From Lemma 2.13 we see that $J$ satisfies $\square \bigvee\left\{B_{\varepsilon}(y) \mid B_{\varepsilon}(y) \in U\right\}$ iff $U \supset_{U} V$ for some $V \in J$, and then $U^{\prime} \supset_{C} V$ for some finite $U^{\prime} \subseteq U$. 
Suppose $J$ satisfies the relations, and $S \in J$ and $\varepsilon \in Q_{+}$. From the relations, find $U \in \mathcal{F} X$ and $V \in J$ such that $\left\{B_{\varepsilon}(x) \mid x \in U\right\} \supset_{U} V$, and then $U^{\prime} \subseteq U$ such that $\left\{B_{\varepsilon}(x) \mid x \in U^{\prime}\right\} \supset_{C} V$ and hence $\left\{B_{\varepsilon}(x) \mid x \in U^{\prime}\right\} \in J$. If $T$ is an upper bound for $S$ and $\left\{B_{\varepsilon}(x) \mid x \in U^{\prime}\right\}$ in $J$, then $\mathrm{r} T<\varepsilon$ as required. The converse argument is essentially the same as for the upper powerlocale.

Notice that although the statement of the Theorem is geometric, its proof is not - it uses frames, suplattices and preframes, which are not geometric structures. However, the proof is topos-valid and so holds at every stage of definition. Implicitly, it also uses the fact that the powerlocale constructions themselves are geometric [32] in order to ensure that Theorem 5.2 applies also to generalized points.

We refine the Theorem slightly to show that $T$ can be chosen of the form $\phi\left(B_{\beta}\left(T^{\prime}\right)\right)$ (with $\phi$ as in Definition 4.4).

Lemma 5.3 Let $X$ be a gms, let $\sim$ stand for $L, U$ or $C$, and suppose $J$ is an ideal of $\left(\mathcal{F}\left(X \times Q_{+}\right), \supset_{\sim}\right)$. If $J$ satisfies the condition of Theorem 5.2, namely that for every $S \in J$ and $\varepsilon \in Q_{+}$there is some $T \in J$ with $S \supset \sim T$ and $\mathrm{r} T<\varepsilon$, then for every $S \in J$ and $\varepsilon \in Q_{+}$there is some $\phi\left(B_{\beta}\left(T^{\prime}\right)\right) \in J$ with $S \supset \sim \phi\left(B_{\beta}\left(T^{\prime}\right)\right)$ and $\beta<\varepsilon$.

Proof We can find $\delta \in Q_{+}$such that $S^{\prime}=\left\{B_{\alpha-\delta}(x) \mid B_{\alpha}(x) \in S\right\} \in J$, and then we can find $T \in J$ such that $S^{\prime} \supset \sim T$ and $r T<\min (\delta, \varepsilon)$. Choose $\beta \in Q_{+}$such that $\mathrm{r} T<\beta<\min (\delta, \varepsilon)$, and let $T^{\prime}=\left\{y \mid \exists \gamma . B_{\gamma}(y) \in T\right\}$. Then $\phi\left(B_{\beta}\left(T^{\prime}\right)\right) \supset_{C} T$, so $\phi\left(B_{\beta}\left(T^{\prime}\right)\right) \in J$. Also, $S \supset \sim \phi\left(B_{\beta}\left(T^{\prime}\right)\right)$. To see this when $\sim$ is L, if $B_{\alpha}(x) \in S$ then we have $B_{\alpha-\delta}(x) \supset B_{\gamma}(y) \in T$, and then $B_{\alpha}(x) \supset B_{\beta}(y) \in \phi\left(B_{\beta}\left(T^{\prime}\right)\right)$, because $\beta-\gamma<\beta<\delta$. The argument when $\sim$ is $\mathrm{U}$ is similar, and then the case for $\mathrm{C}$ follows.

\subsection{Powerlocales of localic completions}

We shall be working with the following diagram. $\phi_{\sim}, \phi_{\sim}^{\prime}$ and the homeomorphism $v_{\sim}^{\prime}$ are Definitions 4.4, 4.7 and 2.12; $v_{\sim}$ is the homeomorphism we are constructing.

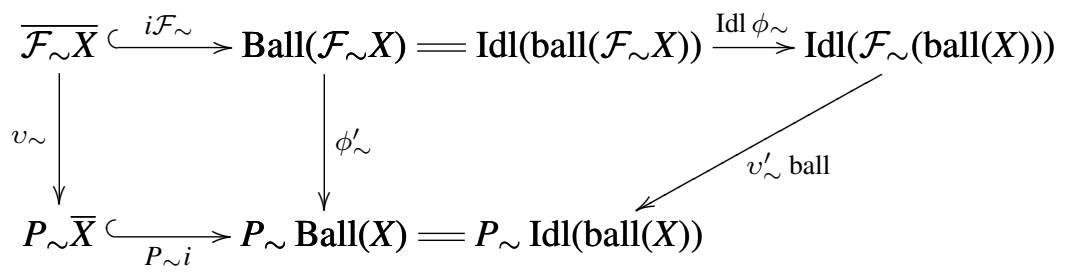


Theorem 5.4 Let $X$ be a gms, and let $\sim$ stand for $L, U$ or $C$. Then $\phi_{\sim}^{\prime}$ factors through a homeomorphism $v_{\sim}: \overline{\mathcal{F}_{\sim} X} \cong P \sim \bar{X}$, giving a monad opfunctor

$\left(\right.$ Comp,$\left.v_{\sim}\right):\left(\operatorname{gms}, \mathcal{F}_{\sim}\right) \rightarrow\left(\operatorname{Loc}, P_{\sim}\right)$ and $i:\left(\right.$ Comp, $\left.v_{\sim}\right) \rightarrow\left(\right.$ Ball, $\left.\phi_{\sim}^{\prime}\right)$ a transformation of monad opfunctors.

Proof Let $I$ be an ideal of $\left(\mathcal{F}_{\sim} X \times Q_{+}, \supset\right)$. We show that if $I$ is Cauchy, then $\operatorname{Idl}\left(\phi_{\sim}\right)(I)=(\supset \sim \phi(I))$ satisfies the condition of Theorem 5.2. Suppose $S \supset \sim$ $\phi\left(B_{\alpha}\left(S^{\prime}\right)\right)$ with $B_{\alpha}\left(S^{\prime}\right) \in I$, and $\varepsilon \in Q_{+}$. We can find $B_{\alpha}\left(S^{\prime}\right) \supset B_{\beta}\left(T^{\prime}\right) \in I$ with $\beta<\varepsilon$, and then, using Lemma 4.5, $S \supset \sim \phi\left(B_{\beta}\left(T^{\prime}\right)\right) \in \operatorname{Idl}\left(\phi_{\sim}\right)(I)$, with $\mathrm{r}\left(\phi\left(B_{\beta}\left(T^{\prime}\right)\right)\right) \leq \beta<\varepsilon$. (We have only $\leq$, because in some situations $T^{\prime}$ might be empty.) Hence $\operatorname{Idl}\left(\phi_{\sim}\right)$ restricts to $v_{\sim}: \overline{\mathcal{F} \sim X} \rightarrow P \sim \bar{X}$.

At this point we see by Lemma 2.7 that (Comp, $v_{\sim}$ ) is a monad opfunctor and $i:\left(\right.$ Comp,$\left.v_{\sim}\right) \rightarrow\left(\right.$ Ball, $\left.\phi_{\sim}^{\prime}\right)$ is a transformation of monad opfunctors. Note that each $P \sim$ preserves embeddings. (In fact this follows from the proof of Theorem 5.2.)

We also have $I=\phi^{-1}\left(\operatorname{Idl}\left(\phi_{\sim}\right)(I)\right)$. For suppose $\phi\left(B_{\delta}(S)\right) \supset \sim \phi\left(B_{\varepsilon}(T)\right)$ with $B_{\varepsilon}(T) \in$ I. By the Cauchy property we can assume $\varepsilon<\delta$, and then by Lemma 4.5 we have $B_{\delta}(S) \supset B_{\varepsilon}(T)$ so $B_{\delta}(S) \in I$.

Now suppose $J$ is an ideal of $\left(\mathcal{F}\left(X \times Q_{+}\right), \supset \sim\right)$ satisfying the condition of Theorem 5.2. We show that $\phi^{-1}(J)$ is an ideal of $\left(\mathcal{F}_{\sim} X \times Q_{+}, \supset\right)$ and that it is Cauchy. Clearly $\phi^{-1}(J)=\left(\supset \phi^{-1}(J)\right)$. For the rest suppose $B_{\alpha_{i}}\left(S_{i}^{\prime}\right) \in \phi^{-1}(J)(i=1,2)$ and $\varepsilon \in Q_{+}$. Then the $\phi\left(B_{\alpha_{i}}\left(S_{i}^{\prime}\right)\right)$ s have an upper bound in the ideal $J$ and using Lemma 5.3 there is some $\phi\left(B_{\beta}\left(T^{\prime}\right)\right) \in J$ such that $\phi\left(B_{\alpha_{i}}\left(S_{i}^{\prime}\right)\right) \supset \sim \phi\left(B_{\beta}\left(T^{\prime}\right)\right)$ and $\beta<\min \left(\alpha_{1}, \alpha_{2}, \varepsilon\right)$. Using Lemma 4.5 we see that $B_{\beta}\left(T^{\prime}\right)$ refines both $B_{\alpha_{i}}\left(S_{i}^{\prime}\right)$ s in $\phi^{-1}(J)$ with $\beta<\varepsilon$, so $\phi^{-1}(J)$ is an ideal and Cauchy. Lemma 5.3 also implies that $J=\left(\supset \sim \phi\left(\phi^{-1}(J)\right)\right)$.

It is straightforward to check that these homeomorphisms restrict to the positive parts, giving $\overline{\mathcal{F}} \underset{\sim}{+} X P_{\sim}^{+} \bar{X}$.

Having identified $P_{\sim} \bar{X}$ with $\overline{\mathcal{F}_{\sim} X}$, we should ask when points of $\overline{\mathcal{F}_{\sim} X}$ are in the and $\diamond$ opens.

Proposition 5.5 Let $B$ be a finite subset of $X \times Q_{+}$.

(1) If $I$ is a Cauchy filter for $\mathcal{F}_{\sim} X(\sim$ standing for $L$ or $C)$, then $v_{\sim}(I)$ is in $\bigwedge\left\{\diamond B_{\delta}(x) \mid(x, \delta) \in B\right\}$ iff there is some $B_{\varepsilon}(S) \in I$ such that $B \supset_{L} \phi\left(B_{\varepsilon}(S)\right)$.

(2) If $I$ is a Cauchy filter for $\mathcal{F}_{\sim} X$ ( standing for $U$ or $C$ ), then $v_{\sim}(I)$ is in $\square(\bigvee B)=\square \bigvee\left\{B_{\delta}(x) \mid(x, \delta) \in B\right\}$ iff there is some $B_{\varepsilon}(S) \in I$ such that $B \supset_{U} \phi\left(B_{\varepsilon}(S)\right)$. 
Proof In each case we consider $I$ as a point of $\operatorname{Ball}\left(\mathcal{F}_{\sim} X\right)$, and ask when $\phi_{\sim}^{\prime}(I)$ is in the corresponding open of $P \sim(\operatorname{Ball}(X))$. The answer can be derived from Lemma 2.13.

(1): $\phi_{\sim}^{\prime}(I)$ is in $\bigwedge\left\{\triangleright B_{\delta}(x) \mid(x, \delta) \in B\right\}$ iff $B \in\left(\supset_{L} \phi_{\sim}^{\prime}(I)\right)=\left(\supset_{L}\left(\supset_{\sim} \phi(I)\right)\right)=\left(\supset_{L}\right.$ $\phi(I))$ where $\phi(I)$ denotes the direct image.

(2) is similar.

Proposition 5.6 Let $I$ be a Cauchy filter for $\mathcal{F}_{C} X$. Then $B_{\delta}(S) \in I$ iff $v_{C}(I)$ is in both $\square\left(\bigvee_{x \in S} B_{\delta}(x)\right)$ and $\bigwedge_{x \in S} \diamond B_{\delta}(x)$.

Proof $\Rightarrow$ is clear. For $\Leftarrow$, and using Proposition 5.5, suppose $\phi\left(B_{\delta}(S)\right) \supset_{U} \phi\left(B_{\alpha}(A)\right)$ and $\phi\left(B_{\delta}(S)\right) \supset_{L} \phi\left(B_{\beta}(B)\right)$ with $B_{\alpha}(A), B_{\beta}(B) \in I$. Choose $B_{\varepsilon}(T) \in I$ such that $B_{\alpha}(A) \supset B_{\varepsilon}(T)$ and $B_{\beta}(B) \supset B_{\varepsilon}(T)$ in $\mathcal{F}_{C} X$, and $\varepsilon<\delta$. Using Lemma 4.5 we have $\phi\left(B_{\delta}(S)\right) \supset \sim \phi\left(B_{\varepsilon}(T)\right)$ with $\sim$ standing for $\mathrm{U}$ or $\mathrm{L}$, and hence also for $\mathrm{C}$. Then again by Lemma 4.5 we have $B_{\delta}(S) \supset B_{\varepsilon}(T)$ in $\mathcal{F}_{C} X$, so $B_{\delta}(S) \in I$.

We complete this section with an analysis of the maps $\Downarrow$ and $\Uparrow$.

Proposition 5.7 For any gms $X$, the identity function on $\mathcal{F} X$ gives non-expansive maps $\mathcal{F}_{C} X \rightarrow \mathcal{F}_{L} X$ and $\mathcal{F}_{C} X \rightarrow \mathcal{F}_{U} X$. These lift to the maps $\Downarrow: P_{C} \bar{X} \rightarrow P_{L} \bar{X}$ and $\Uparrow: P_{C} \bar{X} \rightarrow P_{U} \bar{X}$.

Proof We find monad opfunctors

$$
\begin{aligned}
& \left(\mathbf{g m s}, \operatorname{Id}_{\sim}\right):\left(\mathbf{g m s}, \mathcal{F}_{C}\right) \rightarrow\left(\mathbf{g m s}, \mathcal{F}_{\sim}\right), \\
& \left(\text { cis, } \mathrm{Id}_{\sim}\right):\left(\operatorname{cis}, \mathcal{F}_{C}\right) \rightarrow\left(\text { cis, } \mathcal{F}_{\sim}\right) \text { and } \\
& \left(\text { Loc }, I_{\sim}\right):\left(\text { Loc }, P_{C}\right) \rightarrow\left(\text { Loc }, P_{\sim}\right)
\end{aligned}
$$

where $\mathrm{Id}_{\sim}$ in each case is just the identity on the finite powerset, and $I_{\sim}: P_{C} \rightarrow P_{\sim}$ is $\Uparrow, \Downarrow$ or Id according as $\sim$ is $\mathrm{U}, \mathrm{L}$ or $\mathrm{C}$.

In the following diagram of monad opfunctors, the two squares commute.

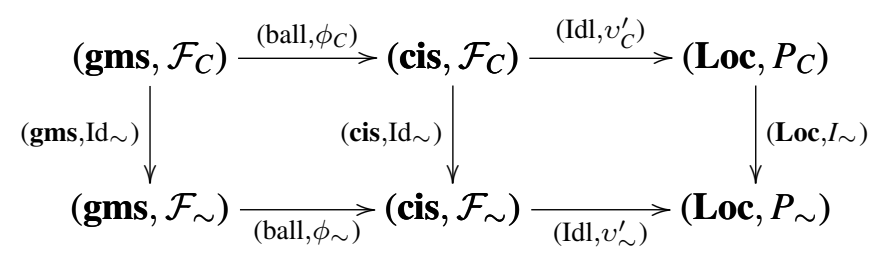

For the left hand square this is elementary, while the right hand can be checked (by considering inverse image functions) using Definition 2.12 - essentially it is because the same definitions work in the different powerlocales. 
These two squares together give a monad opfunctor (Ball, $\left.\psi_{\sim}\right):\left(\mathbf{g m s}, \mathcal{F}_{C}\right) \rightarrow\left(\right.$ Loc,$\left.P_{\sim}\right)$. We have monad opfunctor transformations

$$
\begin{aligned}
& i\left(\mathbf{g m s}, \mathrm{Id}_{\sim}\right):\left(\text { Comp }, v_{\sim}\right)\left(\mathbf{g m s}, \mathrm{Id}_{\sim}\right) \rightarrow\left(\text { Ball }, \psi_{\sim}\right) \text { and } \\
& \quad\left(\mathbf{L o c}, I_{\sim}\right) i:\left(\mathbf{L o c}, I_{\sim}\right)\left(\text { Comp }, v_{C}\right) \rightarrow\left(\text { Ball }, \psi_{\sim}\right) .
\end{aligned}
$$

The identity natural transformation on Comp makes a commutative triangle with these at the level of natural transformations, and we can apply Lemma 2.8 to see that it is an monad opfunctor transformation from $\left(\mathbf{L o c}, I_{\sim}\right)\left(\right.$ Comp,$\left.v_{C}\right)$ to $\left(\right.$ Comp, $\left.v_{\sim}\right)\left(\mathbf{g m s}, \operatorname{Id}_{\sim}\right)$. The effect of this is to show that $\Uparrow$ and $\Downarrow$ are the lifted maps.

\section{Compactness and overtness}

For our first applications we look at two important properties of locales that can be easily addressed using powerlocales.

\subsection{Compactness}

A well known classical property of complete metric spaces $X$ is that they are compact iff totally bounded: for every $\varepsilon>0$ there is a finite $S \subseteq X$ such that the open $\varepsilon$-balls centred on elements of $S$ cover $X$. (Given a dense subspace $D$, one can even choose $S \subseteq D$.) Constructively, [1] used total boundedness as the definition of compactness for complete metric spaces; [18] shows that this is constructively equivalent to compactness of a corresponding localic completion in the style of [33].

We now use powerlocale methods to show this result quite generally for localic completions: a gms completion $\bar{X}$ is compact iff $X$ is totally bounded. (More information on totally bounded quasimetric spaces can be found in [22].) We shall apply a basic result that a locale is compact iff its upper powerlocale has a least point in the following strong sense. We say that a locale $X$ is local if the unique map !: $X \rightarrow 1$ has a left adjoint, a global point $\perp: 1 \rightarrow X$ such that $\perp \circ$ ! $\sqsubseteq \operatorname{Id}_{X}$. This can be expressed by saying that $\perp$ is less than the generic point, in other words that $\perp$ is less than all generalized points. Hence $\perp$ is a bottom point of $X$ in a strong sense. (This is a special case of the concept of local topos, which has been studied in [11]; see [10].) To prove that a locale is local, we shall normally give a geometric definition of the bottom point and show that it is less than every point. The geometricity allows us to deduce that the bottom point is less than the generic point. 
Theorem 6.1 [27]

A locale $Y$ is compact iff $P_{U} Y$ is local. Its bottom point then corresponds to $Y$ as a sublocale of itself.

The usual definition of total boundedness is that for every $\delta>0$ there is a finite subset $S \subseteq X$ that is a $\delta$-cover in the sense that for every $x \in X$ there is some $s \in S$ with $X(s, x)<\delta$. The universal quantification here $(\forall x \in X)$ is intuitionistic but not geometric, so we give a geometric definition in which the relationship between $\delta$ and $S$ is given as a relation Cov in the theory. Hence total boundedness is not (geometrically) a property of a gms but additional structure. We shall, however, present it in such a way that it is unique when it exists.

Definition 6.2 A totally bounded gms $X$ is one equipped with a relation Cov $\subseteq$ $\mathcal{F} X \times Q_{+}$satisfying the following axioms:

(TB1) $\forall S \in \mathcal{F} X . \forall \delta \in Q_{+} \cdot \forall x \in X .(\operatorname{Cov}(S, \delta) \rightarrow \exists s \in S . X(s, x)<\delta)$

$$
\begin{aligned}
& \forall \delta \in Q_{+} \cdot \exists S \in \mathcal{F} X . \operatorname{Cov}(S, \delta) \\
& \forall S \in \mathcal{F} X . \forall \delta \in Q_{+} \cdot\left(\operatorname{Cov}(S, \delta) \rightarrow \exists \delta^{\prime} \in Q_{+} \cdot\left(\delta^{\prime}<\delta \wedge \operatorname{Cov}\left(S, \delta^{\prime}\right)\right)\right. \\
& \forall S, T \in \mathcal{F} X . \forall \delta, \varepsilon \in Q_{+} \cdot\left(\operatorname{Cov}(T, \varepsilon) \wedge \mathcal{F}_{U} X(S, T)<\delta\right. \\
&\rightarrow \operatorname{Cov}(S, \delta+\varepsilon))
\end{aligned}
$$

Note that from TB1 we can deduce that if $\operatorname{Cov}(S, \delta)$ then $\mathcal{F}_{U} X(S, T)<\delta$ for every $T \in \mathcal{F} X$.

Note also that if Cov is viewed (in the obvious way) as a set of formal open balls for $\mathcal{F}_{U} X$, then conditions TB2, TB3 and TB4 are equivalent to saying that Cov is a rounded upper set containing balls of arbitrarily small radius (the Cauchy property).

Proposition 6.3 Let $X$ be a totally bounded gms. Then $\operatorname{Cov}(S, \delta)$ iff $\exists \delta^{\prime}<\delta$ such that $S$ is a $\delta^{\prime}$-cover (ie $\forall x \in X . \exists s \in S . X(s, x)<\delta^{\prime}$ ).

Proof $\Rightarrow$ : Combine TB3 with TB1.

$\Leftarrow$ : By TB2, we can find $T$ with $\operatorname{Cov}\left(T, \delta-\delta^{\prime}\right)$, and by hypothesis $\mathcal{F}_{U} X(S, T)<\delta^{\prime}$. Then $\operatorname{Cov}(S, \delta)$ by TB 4 .

It follows that on any given gms $X$, if Cov can be defined at all then it is unique.

The axiomatization is strong in order to characterize Cov uniquely. However, it is useful to know that we can get by with weaker structure. 
Proposition 6.4 Let $X$ be a gms and let $\operatorname{Cov}_{0} \subseteq \mathcal{F} X \times Q_{+}$be such that axioms TB1 and TB2 hold when $\operatorname{Cov}_{0}$ is substituted for Cov. Then $X$ can be given the structure of total boundedness.

Proof Define $\operatorname{Cov}(S, \delta)$ if for some $\delta^{\prime}<\delta$ and some $T$ we have $\mathcal{F}_{U} X(S, T)<\delta^{\prime}$ and $\operatorname{Cov}_{0}\left(T, \delta-\delta^{\prime}\right)$. We prove the four axioms.

TB1: Suppose $\operatorname{Cov}(S, \delta)$ with $T$ and $\delta^{\prime}$ as above, and suppose $x \in X$. There is some $t \in T$ with $X(t, x)<\delta-\delta^{\prime}$, and then some $s \in S$ with $X(s, t)<\delta^{\prime}$. Then $X(s, x)<\delta$.

TB2: Choose $T$ with $\operatorname{Cov}_{0}(T, \delta / 2)$. We have $\mathcal{F}_{U} X(T, T)=0<\delta / 2$, and it follows that $\operatorname{Cov}(T, \delta)$.

TB3: Given $\operatorname{Cov}(S, \delta)$, in the part of the definition that says $\mathcal{F}_{U} X(S, T)<\delta^{\prime}$ we can reduce $\delta^{\prime}$ to some $\delta^{\prime \prime}$ and thereby reduce $\delta$ to $\delta-\left(\delta^{\prime}-\delta^{\prime \prime}\right)$.

TB4: Given the hypotheses of TB4, we have some $\varepsilon^{\prime}<\varepsilon$ and $U$ such that $\operatorname{Cov}_{0}(U, \varepsilon-$ $\left.\varepsilon^{\prime}\right)$ and $\mathcal{F}_{U} X(T, U)<\varepsilon^{\prime}$. Then $\mathcal{F}_{U} X(S, U)<\delta+\varepsilon^{\prime}$, so $\operatorname{Cov}(S, \delta+\varepsilon)$.

Lemma 6.5 Let $X$ be a totally bounded gms. Then $\overline{\mathcal{F}_{U} X}$ is local, with Cov (as a set of formal balls) its least point.

Proof We have already noted that Cov is upper and Cauchy. For binary filteredness, suppose $\operatorname{Cov}\left(S_{i}, \delta_{i}\right)(i=1,2)$. By TB3 we can find $\delta_{i}^{\prime}<\delta_{i}$ and $\operatorname{Cov}\left(S_{i}, \delta_{i}^{\prime}\right)$. Let $\varepsilon=$ $\min _{i}\left(\delta_{i}-\delta_{i}^{\prime}\right)$ and choose $T$ with $\operatorname{Cov}(T, \varepsilon)$. Then it follows that $\mathcal{F}_{U} X\left(S_{i}, T\right)+\varepsilon<\delta_{i}$, which is what was needed.

Now let $F$ be any point of $\overline{\mathcal{F}_{U} X}$. We want to show $\operatorname{Cov} \subseteq F$. Suppose $\operatorname{Cov}(S, \delta)$, and find $\delta^{\prime}<\delta$ such that $\operatorname{Cov}\left(S, \delta^{\prime}\right)$. By the Cauchy property for $F$, there is some $T$ with $B_{\delta-\delta^{\prime}}(T) \in F$. Then $\mathcal{F}_{U} X(S, T)<\delta^{\prime}$ and so $B_{\delta-\delta^{\prime}}(T) \subset B_{\delta}(S)$ and $B_{\delta}(S) \in F$.

There is a logical subtlety here, relying on the geometric constructivism of the argument. When we let $F$ be "any" point of $\overline{\mathcal{F}_{U} X}$, we allow arbitrary generalized points, not just global points. To explain it in terms of categorical logic, we can use the argument internally in sheaves over $\overline{\mathcal{F}_{U} X}$ to show that Cov is less than the generic point of $\overline{\mathcal{F}_{U} X}$. In other words, the map !; Cov : $\overline{\mathcal{F}_{U} X} \rightarrow 1 \rightarrow \overline{\mathcal{F}_{U} X}$ is less (in the specialization order) than the identity map on $\overline{\mathcal{F}_{U} X}$, which was the definition of locality. This is stronger than saying that $\mathrm{Cov}$ is less than all global points. A more concrete argument can be found by analysing the above proof to find the inverse image function $(! \text { Cov })^{*}$.

Lemma 6.6 Let $X$ be a gms and suppose $\overline{\mathcal{F}_{U} X}$ is local. Then $X$ is totally bounded. 
Proof Let $K$ be the least point of $\overline{\mathcal{F}_{U} X}$. We define $\operatorname{Cov}(S, \delta)$ if $B_{\delta}(S) \in K$. Axioms TB2, TB3 and TB4 follow easily from the fact that $K$ is a Cauchy filter. To prove TB1, suppose $B_{\delta}(S) \in K$ and we are given $x \in X$. Consider the Cauchy filter $\mathcal{Y}(x)=\left\{B_{\varepsilon}(y) \mid X(y, x)<\varepsilon\right\}$. The corresponding point $\uparrow \mathcal{Y}(x)$ of $P_{U} \bar{X} \cong \overline{\mathcal{F}_{U} X}$ is $\left\{B_{\varepsilon}(T) \mid \mathcal{F}_{U} X(T,\{x\})<\varepsilon\right\}$. We have $K \sqsubseteq \uparrow \mathcal{Y}(x)$, and it follows that $\mathcal{F}_{U} X(S,\{x\})<$ $\delta$. Hence there is some $s \in S$ with $X(s, x)<\delta$.

Theorem 6.7 Let $X$ be a gms. Then $\bar{X}$ is compact iff $X$ is totally bounded.

Proof Combine Theorem 6.1 with Lemmas 6.5 and 6.6.

\subsection{Overtness}

"Overt" here is Paul Taylor's synonym of "open" [13], in the sense that a locale $Y$ is overt if the unique map !: $Y \rightarrow 1$ is an open map. This means that for every open $U$ in $Y$, its image under ! is open in 1 and hence is a proposition $\operatorname{Pos}(U)$. Thus Pos is a "positivity predicate". Actually, the positivity predicate can always be defined, by $\operatorname{Pos}(U)$ holding if every open cover of $U$ is inhabited. However [8], overtness implies in addition that every open is a join of positive opens.

Classically, overtness is not an issue since every locale is overt, with $U$ positive iff $U \neq \emptyset$. Constructively it becomes important, and in many ways is a counterpart of compactness. In formal topology, it is often taken as part of the basic definition. (See [17] for a discussion of the relationship between localic overtness and the positivity predicate in formal topology.)

We now use powerlocale methods to provide a simple proof that all gms completions are overt. Dual to localness, a locale $X$ is colocal iff the unique map ! has a right adjoint $T: 1 \rightarrow X$ (a top point). (This is a special case of the concept of totally connected topos [10, C 3.6.16].)

Theorem 6.8 [27]

A locale $Y$ is overt iff $P_{L} Y$ is colocal. Its top point then corresponds to $Y$ as a sublocale of itself.

Corollary 6.9 If $X$ is a gms then $\bar{X}$ is overt.

Proof If $\delta>\varepsilon$ then in $\mathcal{F}_{L} X$ we have $B_{\delta}(S) \supset B_{\varepsilon}(S \cup T)$ for all $S$ and $T$, so any two balls $B_{\delta_{i}}\left(S_{i}\right)(i=1,2)$ have a common refinement $B_{\varepsilon}\left(S_{1} \cup S_{2}\right)$ where $\varepsilon<\min \left(\delta_{1}, \delta_{2}\right)$. It follows that $\mathcal{F} X \times Q_{+}$is a point of $\overline{\mathcal{F}_{L} X}$, and hence must be the top point. We can now apply Theorem 6.8 . 


\section{$7 \quad$ Sublocales of $\mathbb{R}$}

We now look at some applications to those sublocales of the reals $\mathbb{R} \cong \overline{\mathbb{Q}}$ that can be expressed as powerlocale points. Theorem 5.4 offers us the opportunity of defining powerlocale points as Cauchy filters of formal balls, and this gives access to geometric techniques for reasoning with the corresponding sublocales of the reals. The working is reduced to elementary manipulations of the rationals and finite sets of them, and ends up describing subspaces not by their points, but by the opens that contain or meet them. A good example of the style is [38], which deals with connectedness in the reals, with applications to the Intermediate Value Theorem. In fact, as is explained in section 7.1 there, this unfamiliar style of reasoning still makes sense in classical topology. ${ }^{4}$

Since $\mathbb{R}$ is regular, all its sublocales are fitted, and its compact sublocales are closed and hence weakly closed. Thus its powerlocale points can be described as follows.

- In $P_{L} \mathbb{R}$ : overt, weakly closed sublocales.

- In $P_{U} \mathbb{R}$ : compact sublocales.

- In $V \mathbb{R}$ : compact, overt sublocales.

Classically all locales are overt, and weakly closed is equivalent to closed, so it might seem that there is little to choose between the powerlocales. In particular both $P_{U} \mathbb{R}$ and $V \mathbb{R}$ have as their classical points the compact sublocales of $\mathbb{R}$. However, $V \mathbb{R}$ has a finer topology (with opens $\diamond U$ as well as $\square U$ ). Constructively, the requirement for overtness needs extra information on the points. In effect, a compact sublocale is approximated "from above" (the opens $\square U$ provide information about what includes the sublocale), while an overt sublocale is approximated "from below" (the opens $\checkmark U$ provide information about what is in the sublocale). This becomes important in Section 7.1, where we see how to calculate the inf and sup of points of $V^{+} \mathbb{R}$. To approximate $\sup K$ from above or below we need approximations of $K$ from above or below respectively (and inf $K$ is similar but the other way round).

\subsection{Bounds of Vietoris points}

In this section we examine the maps sup and inf from $V^{+} \mathbb{R}$ to $\mathbb{R}$. (We have to use $V^{+} \mathbb{R}$ rather than $V \mathbb{R}$, because the sup and inf of the empty set would have to be infinite.) These can be defined as lifts of gms homomorphisms.

\footnotetext{
${ }^{4}$ Another approach to such questions is Taylor's study [24] in the context of his Abstract Stone Duality. Again, subspaces are described in terms of the opens that contain or meet them.
} 
Proposition 7.1 The functions $\max$ and $\min : \mathcal{F}_{C}^{+} \mathbb{Q} \rightarrow \mathbb{Q}$ are non-expansive.

Proof We prove the result for max. For min it is dual, by order reversal on $\mathbb{Q}$.

Let $S$ and $T$ be in $\mathcal{F}^{+} \mathbb{Q}$, let $s_{\max }=\max (S), t_{\max }=\max (T)$, and let $q=\mathcal{F}_{C}^{+} \mathbb{Q}(S, T)$, a non-negative rational. Since $t_{\max } \in T$ we have that $t_{\max }$ is within $q$ of some $s \in S$ and then $t_{\max } \leq s+q \leq s_{\max }+q$. Similarly, $s_{\max } \leq t_{\max }+q$ and so $\left|t_{\max }-s_{\max }\right| \leq q$.

Definition 7.2 We define sup: $V^{+} \mathbb{R} \rightarrow \mathbb{R}$ as $\overline{\max }: V^{+} \mathbb{R} \cong \overline{\mathcal{F}_{C}^{+} \mathbb{Q}} \rightarrow \overline{\mathbb{Q}} \cong \mathbb{R}$. Similarly, inf is $\min$.

Our goal now is to show how, if $K$ is a point of $V^{+} \mathbb{R}, \sup (K)$ genuinely is the supremum of $K$. (We focus on sup from now on; the results for inf are entirely dual.) We show in fact that it is the greatest element of $K: \sup (K) \in K$, and if $x \in K$ (see Theorem 2.3) then $x \leq \sup (K)$.

Lemma 7.3 If $K$ is a point of $V^{+} \mathbb{R}$, then $\sup (K) \in K$.

Proof We must show $\downarrow \sup (K) \sqsubseteq \Downarrow K$ and $\uparrow \sup (K) \sqsupseteq \Uparrow K$. Now sup; $\downarrow: V^{+} \mathbb{R} \rightarrow$ $\mathbb{R} \rightarrow P_{L} \mathbb{R}$ lifts $\max ;\{-\}: \mathcal{F}_{C}^{+} \mathbb{Q} \rightarrow \mathbb{Q} \rightarrow \mathcal{F}_{L} \mathbb{Q}$ and by Proposition $5.7 \Downarrow: V^{+} \mathbb{R} \rightarrow$ $P_{L} \mathbb{R}$ lifts Id: $\mathcal{F}_{C}^{+} \mathbb{Q} \rightarrow \mathcal{F}_{L} \mathbb{Q}$. Hence to prove $\downarrow$ osup $\sqsubseteq \Downarrow$ it suffices by Proposition 2.21 to show that $\mathcal{F}_{L} \mathbb{Q}(\{\max (S)\}, S)=0$ for every $S \in \mathcal{F}^{+} \mathbb{Q}$. This is obvious, because $\max (S) \in S$. The other half, $\uparrow \circ \sup \sqsupseteq \Uparrow$, is dual.

Lemma 7.4 If $x$ is a point of $\mathbb{R}$ then $\sup (\{x\})=x$.

Proof $\{-\}$; sup: $\mathbb{R} \rightarrow V^{+} \mathbb{R} \rightarrow \mathbb{R}$ lifts $\{-\} ; \max : \mathbb{Q} \rightarrow \mathcal{F}_{C}^{+} \mathbb{Q} \rightarrow \mathbb{Q}$, which is the identity.

We now proceed to show that if $K \subseteq L$ are points of $V^{+} \mathbb{R}$, then $\sup (K) \leq \sup (L)$. Note that by $K \subseteq L$ we mean the order corresponding to that of the sublocales: $\Uparrow K \sqsupseteq \Uparrow L$ and $\Downarrow K \sqsubseteq \Downarrow L$.

We first investigate a locale that will be useful.

Definition 7.5 Let $\overrightarrow{\mathbb{Q}}$ be the gms whose elements are the rationals, but whose metric is defined as truncated minus,

$$
\overrightarrow{\mathbb{Q}}(x, y)=x \dot{-} y=\max (0, x-y) .
$$


It is shown in [33] that $\overline{\overrightarrow{\mathbb{Q}}}$ is homeomorphic to the ideal completion of $(\mathbb{Q},<)$, in other words its points are equivalent to rounded lower inhabited subsets of $\mathbb{Q}$. It is the locale $\overrightarrow{(-\infty, \infty]}$ of lower reals.

Lemma 7.6 In $\overrightarrow{\mathbb{Q}}$ we have $B_{\varepsilon}(y) \subset B_{\delta}(x)$ iff $\varepsilon<\delta$ and $x-\delta<y-\varepsilon$.

Proof

$$
\begin{aligned}
B_{\varepsilon}(y) \subset B_{\delta}(x) & \Leftrightarrow(x \dot{-} y)<\delta-\varepsilon \\
& \Leftrightarrow 0<\delta-\varepsilon \text { and } x-y<\delta-\varepsilon
\end{aligned}
$$

Lemma 7.7 $\mathrm{Id}: \mathbb{Q} \rightarrow \overrightarrow{\mathbb{Q}}$ lifts to the map $\downarrow: \mathbb{R} \rightarrow \overrightarrow{(-\infty, \infty]}$ that takes each Dedekind section $(L, R)$ to $L$.

Proof It is shown in [33] that $(L, R)$ as a Cauchy filter is

$$
\left\{B_{\delta}(x) \mid x-\delta \in L, x+\delta \in R\right\} .
$$

This maps to

$$
\left\{B_{\varepsilon}(y) \mid \exists x, \delta .(x-\delta \in L \text { and } x+\delta \in R \text { and } \delta<\varepsilon \text { and } y-\varepsilon<x-\delta)\right\}
$$

and this in turn corresponds, as an ideal of $(\mathbb{Q},<)$, to

$$
\{y-\varepsilon \mid \exists x, \delta .(x-\delta \in L \text { and } x+\delta \in R \text { and } \delta<\varepsilon \text { and } y-\varepsilon<x-\delta)\} .
$$

But this is just $L$ again. To show that it contains $L$, suppose $z \in L$, and by roundedness find $z<z^{\prime} \in L$. Now find $w \in R$ and let $x=\left(z^{\prime}+w\right) / 2, \delta=\left(w-z^{\prime}\right) / 2=x-z^{\prime}$. Then $x-\delta=z^{\prime} \in L$ and $x+\delta=w \in R$. Let $y=x, \varepsilon=y-z>\delta$. Then $y-\varepsilon=z<z^{\prime}=x-\delta$.

Lemma 7.8 If $K \subseteq L$ are points of $V^{+} \mathbb{R}$, then $\sup (K) \leq \sup (L)$.

Proof The function max: $\mathcal{F}_{L}^{+} \mathbb{Q} \rightarrow \overrightarrow{\mathbb{Q}}$ is non-expansive. Let $S, T \in \mathcal{F}^{+} \mathbb{Q}, s_{\max }=$ $\max (S), t_{\max }=\max (T)$ and $q=\mathcal{F}_{L}^{+} \mathbb{Q}(S, T) \geq 0$. Since $s_{\max } \in S$ we can find $t \in T$ within $q$ of $s_{\max }$, and then $s_{\max } \leq t+q \leq t_{\max }+q$ and $s_{\max } \dot{-} t_{\max } \leq q$. 
Using Proposition 5.7 and Lemma 7.7, the first of the following two commutative squares lifts to the second.
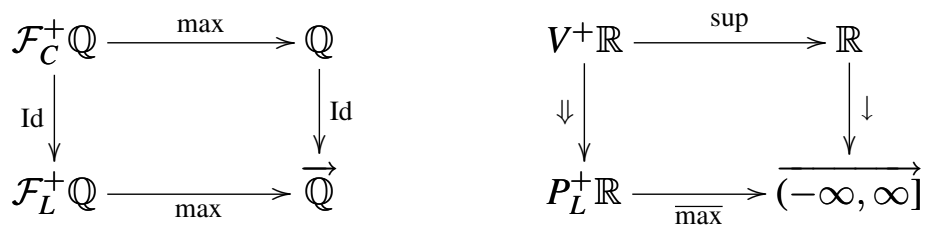

Since $K \subseteq L$ we have $\Downarrow K \sqsubseteq \Downarrow L$, and it follows that $\downarrow \sup (K) \sqsubseteq \downarrow \sup (L)$. This says that $\sup (L)$ has the larger left half in its Dedekind section, in other words that $\sup (K) \leq \sup (L)$.

We can similarly show that $\inf (K) \geq \inf (L)$, but this time the proof has to use the upper powerlocale.

The proofs show something of the reason why we need points of the Vietoris powerlocale if we are to calculate sup and inf. Given a point of the lower powerlocale, we can approximate its sup from below but not from above. This gives a point of $\overrightarrow{(-\infty, \infty]}$, which in fact is what $\overline{\max }$ calculates in the above proof. Similarly, we can approximate its inf from above but not below, getting a point in the dual $[-\infty, \infty)$ (completing the dual metric $\overleftarrow{\mathbb{Q}}(x, y)=y \dot{-} x)$. The same applies to points of the upper powerlocale, but the other way round. To get sup and inf as full Dedekind sections, approximated from both below and above, we need to start with a point of the Vietoris powerlocale.

Putting all these together, we obtain

Theorem 7.9 There are maps sup, inf : $V^{+} \mathbb{R} \rightarrow \mathbb{R}$ such that $\sup (K)$ is the greatest point in $K$ and $\inf (K)$ is the least.

\subsection{The Heine-Borel maps}

If $x \leq y$ are reals then the Heine-Borel Theorem says that the closed interval $[x, y]$ is compact. As it happens, it is also overt and semifitted, and so corresponds to a point $H B_{C}(x, y)$ of $V^{+} \mathbb{R}$. In fact, this gives a continuous map $H B_{C}: \leq \rightarrow V^{+} \mathbb{R}$. To see continuity, first note that if $U$ is open, then $[x, y] \subseteq U$ iff there is some rational open interval $(p, q) \leq U$ containing both $x$ and $y$. Hence $H B_{C}^{-1}(\square U)=$ $\bigvee\{(p, \infty) \times(-\infty, q) \mid p<q,(p, q) \leq U\}$. Next, $[x, y]$ meets $U$ iff there is some 
rational open interval $(p, q) \leq U$ such that $x<q$ and $p<y$, so $H B_{C}^{-1}(\diamond U)=$ $\bigvee\{(-\infty, q) \times(p, \infty) \mid p<q,(p, q) \leq U\}$

We shall turn this argument on its head. Suppose we can define a map $H B_{C}: \leq \rightarrow$ $V^{+} \mathbb{R}$, and show that $H B_{C}(x, y)$ corresponds to the subspace $[x, y]$ for reals $x \leq y$. Then we have shown that $[x, y]$ is always compact.

Remark 7.10 $[0,1]$ is homeomorphic to $\overline{(0,1) \cap \mathbb{Q}}$, after which its compactness follows by Theorem 6.7 from the fact that $(0,1) \cap \mathbb{Q}$ is totally bounded. A similar technique works for other closed intervals. In fact in the case where $x<y$ we have $[x, y] \cong[0,1]$. Hence our metric space techniques already give a proof of the localic Heine-Borel.

The paper [38] (written as a sequel to the present paper, though published earlier) shows that $H B_{C}$ factors via a new powerlocale $V^{c} \mathbb{R}$ whose points are connected points of $V^{+} \mathbb{R}$ (in fact it gives a homeomorphism $\leq \cong V^{c} \mathbb{R}$ ). It exploits $H B_{C}$ in its discussion of the Intermediate Value Theorem and Rolle's Theorem.

To define $H B_{C}$ it will be convenient also to use two simpler maps $H B_{L}=\Downarrow \circ H B_{C}$ and $H B_{U}=\Uparrow \circ H B_{C}$, taking their values in $P_{L} \mathbb{R}$ and $P_{U} \mathbb{R}$.

To find the closed interval $[x, y]$ as a point of a powerlocale, our working in effect requires us to define the "upper" and "lower" distances from $S$ to $[x, y]$ for every finite $S \subseteq \mathbb{Q}$. In classical terms these would appear as $\sup _{z \in[x, y]} \min _{s \in S} d(s, z)$ and $\max _{s \in S} \inf _{z \in[x, y]} d(s, z)$. The upper distance is less than $q$ iff every $z$ in $[x, y]$ is within $q$ of some $s$ in $S$, in other words $\left\{B_{q}(s) \mid s \in S\right\}$ covers $[x, y]$. The lower distance is less than $q$ iff every $s$ in $S$ is in the interval $(x-q, y+q)$. However, we must express these geometrically.

Definition 7.11 Let $x \leq y$ be reals, and let $S$ be a non-empty finite subset of $\mathbb{Q} \times Q_{+}$. We say that $\left\{B_{\varepsilon}(s) \mid(s, \varepsilon) \in S\right\}$ covers $[x, y]$ if there is some non-empty finite sequence $\left(s_{i}, \varepsilon_{i}\right)(1 \leq i \leq n)$ in $S$ such that

$$
\begin{aligned}
s_{1}-\varepsilon_{1} & <x \\
s_{i+1}-\varepsilon_{i+1} & <s_{i}+\varepsilon_{i} \quad(1 \leq i<n) \\
y & <s_{n}+\varepsilon_{n}
\end{aligned}
$$

It is simpler for us not to assume that $s_{1} \leq s_{2} \leq \cdots \leq s_{n}$. It could be that the ball $B_{\varepsilon_{i+1}}\left(s_{i+1}\right)$ is a long way to the left of $B_{\varepsilon_{i}}\left(s_{i}\right)$ with a big gap in between. For present purposes it turns out not to matter, though [38] also shows that a better behaved subsequence can always be found. 
Lemma 7.12 Suppose $\left\{B_{\varepsilon}(s) \mid(s, \varepsilon) \in S\right\}$ covers $[x, y]$ with a sequence $\left(s_{i}, \varepsilon_{i}\right)$ as described. Suppose also $x \leq z \leq y$. Then for some $i$ we have $s_{i}-\varepsilon_{i}<z<s_{i}+\varepsilon_{i}$.

Proof The sequence also covers $[x, z]$, for $z \leq y<s_{n}+\varepsilon_{n}$ implies $z<s_{n}+\varepsilon_{n}$. (Proof: choose a rational $q$ such that $y<q<s_{n}+\varepsilon_{n}$. Then we have either $z<s_{n}+\varepsilon_{n}$, as desired, or $q<z$. But this second alternative implies $y<z$, which is impossible.) Hence without loss of generality we can assume that $z=y$. By a similar argument we can furthermore assume that $x=z$ and the closed interval is a single point. We use induction on $n$. If $n=1$ then we have $s_{1}-\varepsilon_{1}<z<s_{1}+\varepsilon_{1}$ and we are done. Now suppose $n>1$. Since $s_{n}-\varepsilon_{n}<s_{n-1}+\varepsilon_{n-1}$, we have either $s_{n}-\varepsilon_{n}<z$, in which case we can take $i=n$, or $z<s_{n-1}+\varepsilon_{n-1}$, in which case we can use induction.

It is clear that if we have a cover and we enlarge all the balls then we still have a cover; and also that we are able to shrink the balls slightly and still have a cover.

Recall the natural transformations $\phi_{\sim}$ from Definition 4.4.

Definition 7.13 Let $x \leq y$ be reals. Then we define subsets $\mathrm{HB}_{\sim}(x, y)$ of $\mathcal{F}^{+} \mathbb{Q} \times Q_{+}$ by

$$
\begin{aligned}
B_{\delta}(S) & \in \operatorname{HB}_{U}(x, y) \text { iff } \phi\left(B_{\delta}(S)\right) \text { covers }[x, y] \\
B_{\delta}(S) & \in \operatorname{HB}_{L}(x, y) \text { iff } \forall s \in S .(x<s+\delta \wedge s-\delta<y) \\
\operatorname{HB}_{C}(x, y) & =\operatorname{HB}_{U}(x, y) \cap \operatorname{HB}_{L}(x, y)
\end{aligned}
$$

The condition for $\operatorname{HB}_{L}(x, y)$ is stating that each $B_{\delta}(s)$ overlaps $[x, y]$ : for it would fail to overlap iff $s+\delta \leq x$ or $y \leq s-\delta$.

Lemma 7.14 Suppose $x \leq y$ are reals, $S, T \in \mathcal{F}^{+} \mathbb{Q}, B_{\delta}(S) \in \operatorname{HB}_{U}(x, y)$ and $B_{\varepsilon}(T) \in \operatorname{HB}_{L}(x, y)$. Then

$$
\mathcal{F}_{U} \mathbb{Q}(S, T)=\mathcal{F}_{L} \mathbb{Q}(T, S)<\delta+\varepsilon .
$$

Proof The first equality is immediate from the symmetry of our gms structure on $\mathbb{Q}$. If $t \in T$ then $t$ is in the open interval $(x-\varepsilon, y+\varepsilon)$. However, the closed interval $[x-\varepsilon, y+\varepsilon]$ is covered by $\left\{B_{\delta+\varepsilon}(s) \mid s \in S\right\}$, and so by Lemma 7.12 there is some $s \in S$ with $s-\delta-\varepsilon<t<s+\delta+\varepsilon$. Hence $\mathcal{F}_{U} \mathbb{Q}(S, T)<\delta+\varepsilon$.

Lemma 7.15 Let $x \leq y$ be reals and let $\varepsilon \in Q_{+}$. Then there is some $S \in \mathcal{F}^{+} \mathbb{Q}$ such that $B_{\varepsilon}(S) \in \mathrm{HB}_{C}(x, y)$. 
Proof Choose rationals $a$ and $b$ with $a<x<a+\varepsilon$ and $b-\varepsilon<y<b$. Now choose some natural number $k \geq 1$ such that $\mu<2 \varepsilon$, where $\mu=(b-a) / k$. If we divide the interval $(a, b)$ into $k$ equal parts, each of length $\mu$, then their centres are

$$
s_{i}=a+(2 i-1) \mu / 2(1 \leq i \leq k) .
$$

Let $S=\left\{s_{i} \mid 1 \leq i \leq k\right\}$.

Lemma 7.16 Suppose $x \leq y$ are reals, and suppose (with $\sim$ being either $U, L$ or $C$ ) we have

$$
B_{\delta_{\lambda}}\left(S_{\lambda}\right) \in \mathrm{HB}_{\sim}(x, y) \quad(\lambda=1,2) .
$$

Then there is some $B_{\varepsilon}(T) \in \mathrm{HB}_{C}(x, y)$ such that $\mathcal{F}_{\sim} \mathbb{Q}\left(S_{\lambda}, T\right)+\varepsilon<\delta_{\lambda}$.

Proof We can find $\varepsilon$ such that $B_{\delta_{\lambda}-2 \varepsilon}\left(S_{\lambda}\right) \in \mathrm{HB}_{\sim}(x, y)$ for $\lambda=1,2$. By Lemma 7.15 we can find $T$ with $B_{\varepsilon}(T) \in \operatorname{HB}_{C}(x, y)$. Now by Lemma 7.14 if $\sim$ is $\mathrm{U}$ or $\mathrm{L}$ we deduce

$$
\mathcal{F}_{\sim} \mathbb{Q}\left(S_{\lambda}, T\right)<\delta_{\lambda}-2 \varepsilon+\varepsilon=\delta_{\lambda}-\varepsilon .
$$

The case when $\sim$ is $\mathrm{C}$ follows.

Proposition 7.17 The definitions above define maps $\mathrm{HB}_{\sim}: \quad \leq \rightarrow \overline{\mathcal{F}_{\sim}^{+} \mathbb{Q}}$ (with $\sim$ being $U, L$ or $C$ ).

Proof We must show that $H B_{\sim}(x, y)$ is a Cauchy filter. Upper closedness is obvious, and binary filteredness and the Cauchy property follow from Lemmas 7.16 and 7.15.

Lemma 7.18 Let $x \leq y$ be reals. Then

(1) $\Downarrow \mathrm{HB}_{C}(x, y)=\mathrm{HB}_{L}(x, y)$ and

(2) $\Uparrow \mathrm{HB}_{C}(x, y)=\mathrm{HB}_{U}(x, y)$.

Proof By Proposition 5.7 and Definition 2.20 we must show that (with $\sim$ standing for $\mathrm{L}$ or $\mathrm{U}) H B_{\sim}(x, y)=\left(\supset H B_{C}(x, y)\right)$, where $\supset$ is the ball refinement of $\mathcal{F}_{\sim} \mathbb{Q}$. This follows from Lemma 7.16.

Theorem 7.19 If $x \leq y$ are reals, then $\mathrm{HB}_{\sim}(x, y)$, as point of $P_{\sim} \mathbb{R}$, corresponds to the closed interval sublocale $[x, y]$. 
Proof When $\sim$ is $\mathrm{U}$ : We show that a point $z$ of $\mathbb{R}$ has $\uparrow z \sqsupseteq \operatorname{HB}_{U}(x, y)$ iff $x \leq z \leq y$. We have that $\uparrow z \sqsupseteq \operatorname{HB}_{U}(x, y)$ iff whenever $\left\{B_{\varepsilon}(s) \mid s \in S\right\}$ covers $[x, y]$ then $z$ is in $B_{\varepsilon}(s)$ for some $s$ in $S$. That this is implied by $x \leq z \leq y$ has already been proved in Lemma 7.12. For the converse we wish to show $x \leq z$, ie that $(x, z)$ is in the closed complement of the open sublocale $>$ of $\mathbb{R}^{2}$, so suppose $z<q<x$ for some rational $q$. Choosing also a rational $r>y$ such that $r>q$, the ball $B_{(r-q) / 2}((q+r) / 2)$ covers $[x, y]$ but does not contain $z$. Similarly, $z \leq y$.

When $\sim$ is L: We show that a point $z$ of $\mathbb{R}$ has $\downarrow z \sqsubseteq \operatorname{HB}_{L}(x, y)$ iff $x \leq z \leq y$. We have that $\downarrow z \sqsubseteq \operatorname{HB}_{L}(x, y)$ iff whenever $s-\varepsilon<z<s+\varepsilon$ then $x<s+\varepsilon$ and $s-\varepsilon<y$. This is obviously implied by $x \leq z \leq y$. For the converse, if $z<x$ then we can find rationals $q$ and $r$ with $q<z<r<x$ and then by taking $s=q$ and $\varepsilon=r-q$ we get a contradiction. Hence $x \leq z$, and similarly $z \leq y$.

When $\sim$ is C: For any locale $X$, the sublocale corresponding to a point $K$ of $V X$ is the sublocale meet of those corresponding to $\Uparrow K$ and $\Downarrow K$. Hence by Lemma 7.18, the sublocale corresponding to $\mathrm{HB}_{C}(x, y)$ is the meet of those for $\mathrm{HB}_{U}(x, y)$ and $\mathrm{HB}_{L}(x, y)$, so it is just $[x, y]$ again.

Corollary 7.20 (Heine-Borel Theorem) If $x$ and $y$ are reals, then the closed interval $[x, y]$ is compact.

Proof This follows already from part (U) of the Theorem 7.19, since the points of $P_{U} \mathbb{R}$ are equivalent to compact fitted sublocales of $\mathbb{R}$.

Example 7.21 Let $f: \mathbb{R} \rightarrow \mathbb{R}$ be a map. Then we can define $\sup _{x \leq z \leq y} f(z)$ (as a continuous function of $x$ and $y$ ) localically as the composite map

$$
H B_{C} ; V^{+} f ; \sup : \quad \leq \rightarrow V^{+} \mathbb{R} \rightarrow V^{+} \mathbb{R} \rightarrow \mathbb{R} .
$$

The main technical question here is how we know $V^{+} f$ takes points of $V^{+} \mathbb{R}$ to their sublocale images under $f$. This is discussed in more detail in [38].

Remark 7.22 We have defined $H B_{\sim}(x, y)$ in the case where $x \leq y$. One might consider it natural to extend this so that $H B_{\sim}(x, y)$ is empty if $x>y$, but a simple argument shows this is impossible for $H B_{L}$ and $H B_{C}$. Consider the point $\emptyset$. It is bottom in $P_{L} X$ and isolated in $V X$, and in each case $\{\emptyset\}$ is closed. Hence $H B_{L}^{-1}(\{\emptyset\})$ and $H B_{C}^{-1}(\{\emptyset\})$ would also have to be closed; but our ambition was to have them equal to $>$, which is open but not closed.

In $P_{U} X$ on the other hand, $\emptyset$ is the top point and $\{\emptyset\}$ is open. We sketch a modified construction of $H B_{U}$ that extends it to a map $\mathbb{R} \times \mathbb{R} \rightarrow \overline{\mathcal{F}_{U} \mathbb{Q}}$ in the way suggested. 
We generalize the previous definition of "covers" to say that, for $S$ a finite subset of $\mathbb{Q} \times Q_{+},\left\{B_{\varepsilon}(s) \mid(s, \varepsilon) \in S\right\}$ covers $[x, y]$ iff either $x>y$ or it has a non-empty finite sequence with the property described before. (Note that in this second case we still do not assume $x \leq y$.)

The proof of Proposition 7.17 must now be modified slightly. For the Cauchy property, if $\varepsilon \in Q_{+}$then we find rationals $a$ and $b$ with $a<x$ and $b>y$. Without loss of generality we can suppose $a<b$. By Lemma 7.15 we can then find $U$ with $B_{\varepsilon}(U) \in \mathrm{HB}_{C}(a, b) \subseteq \mathrm{HB}_{U}(a, b) \subseteq \mathrm{HB}_{U}(x, y)$.

For binary filteredness, suppose $B_{\delta_{\lambda}}\left(S_{\lambda}\right) \in \mathrm{HB}_{U}(x, y)(\lambda=1,2)$. We can find $\varepsilon^{\prime} \in Q_{+}$ with $B_{\delta_{\lambda}-2 \varepsilon^{\prime}}\left(S_{\lambda}\right) \in \mathrm{HB}_{U}(x, y)$. We shall now find $B_{\varepsilon}(T)$ such that $B_{\varepsilon}(T) \in \operatorname{HB}_{U}(x, y)$ and $\mathcal{F}_{U} \mathbb{Q}\left(S_{\lambda}, T\right)+\varepsilon<\delta_{\lambda}$. If $x>y$ then we can choose $T=\emptyset$ and $\varepsilon=\varepsilon^{\prime}$. In the other case, we have covering sequences $\left(\left(s_{i}^{\lambda}, \delta_{\lambda}-2 \varepsilon^{\prime}\right)\right)_{1 \leq i \leq m_{\lambda}}$ taken from $S_{\lambda}$. Let

$$
\begin{aligned}
& a=\max _{\lambda}\left(s_{1}^{\lambda}-\delta_{\lambda}+2 \varepsilon^{\prime}\right)<x \\
& b=\min _{\lambda}\left(s_{m_{\lambda}}^{\lambda}+\delta_{\lambda}-2 \varepsilon^{\prime}\right)>y .
\end{aligned}
$$

Note that $B_{\delta_{\lambda}-\varepsilon^{\prime}}\left(S_{\lambda}\right) \in \operatorname{HB}_{U}(a, b)$. If $b<a$ then $y<x$, so (since the order is decidable on $\mathbb{Q}$ ) we can assume $a \leq b$, and then by Lemma 7.16 we can find

$$
B_{\varepsilon}(T) \in \operatorname{HB}_{C}(a, b) \subseteq \operatorname{HB}_{U}(a, b) \subseteq \mathrm{HB}_{U}(x, y)
$$

such that

$$
\mathcal{F}_{U} \mathbb{Q}\left(S_{\lambda}, T\right)+\varepsilon<\delta_{\lambda}-\varepsilon^{\prime}<\delta_{\lambda}
$$

Martín Escardó has pointed out that in some situations it is useful to precompose $H B \sim$ with $\langle\min , \max \rangle: \mathbb{R} \times \mathbb{R} \rightarrow \leq$, thus recovering a map with domain $\mathbb{R} \times \mathbb{R}$. (This does not recover the $H B_{U}$ defined above, of course.)

\section{Conclusions}

We have presented a constructive, localic account of hyperspace techniques for dealing with certain kinds of subspaces of complete metric spaces. Our account is very general, covering localic completions of generalized metric spaces in the sense of Lawvere [14], and it uses that generality to include the lower and upper powerlocales (localic hyperspaces) where the metric is necessarily asymmetric. The techniques are computationally convenient, as illustrated by the applications here. As applied to compact intervals in the reals, they have been extended [38] to discuss connectedness 
and give constructive localic accounts of the Intermediate Value Theorem and Rolle's Theorem.

Our results are analogues of existing classical, spatial results ([2], [3], [20]). [2] discusses powerdomains for completions of generalized metric spaces, and shows that they too are completions of spaces of finite subsets. However, their classical proofs are based on a definition of completion as closure by limits within a space of modules. Our constructive, localic results, though clearly analogous, are quite different in their techniques and technical content even when viewed in terms of points. Our completion (from [33]) is defined with a direct representation of the points as Cauchy filters, and for our analogues of the powerdomains defined in [2] we have been able to exploit the established theory of powerlocales.

One constructivist aspect of the results is that they indicate how to get good predicative tools for dealing with these powerlocales. The definition of powerlocale uses the frame of opens of $X$ as a set of generators for $P_{U} X$ or $P_{L} X$, and on the face of it this underlay our treatment particularly in Section 5.1. This is topos-valid, but not predicative, since the construction of the frame of opens requires the powerset axiom. However, there are predicative approaches for extracting generators and relations for powerlocales out of those for the original locale, and some of these are set out (in the context of predicative formal topology) in [35] and [34]. In fact, our proofs in Section 5.1 could be made compatible with that development. When translated thus into predicative mathematics the general results can be complicated to use. We have here transformed them into a special form that applies in the case of gms completion and naturally extends conventional ideas using the Hausdorff metric.

Finally, it is natural to wonder whether the technical development can be simplified, perhaps avoiding the lengthy detour via continuous dcpos and the ball domain, or perhaps making better use of the pointwise reasoning techniques (in a point-free setting) of geometric logic. I would hope it can, but in the 10 years since [29] was issued I have not managed to simplify the overall argument despite having tidied the proofs considerably. Nonetheless, the use of ball domains does show connections with a technique (see [3]) that has already had some success in relating computation and domain theory to metric spaces. As for the pointwise reasoning, this seems to call for better ideas than have so far come to me. Even for the particular case of $V \mathbb{R}$, the classical equivalence between compact subspaces and points of $\overline{\mathcal{F}_{C} \mathbb{Q}}$ (see [38]) is intricate and does not seem to have an analogue in geometric reasoning.

Acknowledgements In its early stages this work was conducted with the support of the Engineering and Physical Sciences Research Council through the project Foun- 
dational Structures in Computer Science at the Department of Computing, Imperial College. The author is very grateful to Prof. Edmund Robinson, whose careful criticism of an earlier version stimulated some fundamental revision of the exposition and technical content (in particular, a better integration of the accounts for the three powerlocales and closer attention to the monad structure) and of the discussion of the relation to previous work such as [3], [2] and [20]. The author also thanks two anonymous referees for their useful comments.

\section{References}

[1] E Bishop, D Bridges, Constructive Analysis, Springer-Verlag, 1985.

[2] M Bonsangue, F van Breugel, J Rutten, Generalized Metric Spaces: Completion, Topology, and Powerdomains via the Yoneda Embedding, Theoret. Comput. Sci. 193 (1998), 1-51; doi:10.1016/S0304-3975(97)00042-X.

[3] A Edalat, R Heckmann, A Computational Model for Metric Spaces, Theoret. Comput. Sci. 193 (1998), 53-73; doi:10.1016/S0304-3975(96)00243-5.

[4] M Fourman, J Hyland, Sheaf Models for Analysis, from: "Applications of Sheaves", (M Fourman, C Mulvey, D Scott, editors), Lecture Notes in Mathematics 753, SpringerVerlag (1979), 280-301; doi:10.1007/BFb0061823.

[5] G Gierz, K Hofmann, K Keimel, J Lawson, M Mislove, D Scott, A Compendium of Continuous Lattices, Springer-Verlag, 1980.

[6] K H Hofmann, M W Mislove, Local Compactness and Continuous Lattices, from: "Continuous Lattices: Proceedings, Bremen, 1979", (B Banaschewski, R-E Hoffmann, editors), Lecture Notes in Mathematics 871, Springer-Verlag, 1981, 209-248; doi:10.1007/BFb0089908.

[7] P Johnstone, Stone Spaces, Cambridge Studies in Advanced Mathematics 3, Cambridge University Press, 1982.

[8] P Johnstone, Open Locales and Exponentiation, Contemporary Mathematics 30 (1984), 84-116.

[9] P Johnstone, Vietoris Locales and Localic Semi-lattices, from: "Continuous Lattices and their Applications”, (R-E Hoffmann, editor), Pure and Applied Mathematics 101, Marcel Dekker, 1985, 155-18.

[10] P Johnstone, Sketches of an Elephant: A Topos Theory Compendium, vol. 2, Oxford Logic Guides 44, Oxford University Press, 2002.

[11] P Johnstone, I Moerdijk, Local Maps of Toposes, Proc. Lond. Math. Soc. (3rd series) 58 (1989), 281-305; doi:10.1112/plms/s3-58.2.281. 
[12] P Johnstone, S Vickers, Preframe Presentations Present, from: "Category Theory - Proceedings, Como 1990”, (A Carboni, M Pedicchio, G Rosolini, editors), Lecture Notes in Mathematics 1488, Springer-Verlag, 1991, 193-212; doi:10.1007/BFb0084221.

[13] A Joyal, M Tierney, An Extension of the Galois Theory of Grothendieck, Memoirs of the American Mathematical Society 309 (1984).

[14] F Lawvere, Metric Spaces, Generalized Logic, and Closed Categories, Rend. del Sem. Mat. e Fis. di Milano 43 (1973). Reissued as [15]. doi:10.1007/BF02924844.

[15] F Lawvere, Metric Spaces, Generalized Logic, and Closed Categories, Reprints in Theory and Applications of Categories 1 (2002), 1-37. Originally published as [14].

[16] S Mac Lane, Categories for the Working Mathematician, Springer-Verlag, 1971.

[17] S Negri, Continuous Domains as Formal Spaces, Mathematical Structures in Computer Science 12 (2002), 19-52; doi:10.1017/S0960129501003450.

[18] E Palmgren, A Constructive and Functorial Embedding of Locally Compact Metric Spaces into Locales, Topology and its Applications 154 (2007), 1854-1880; doi:10.1016/j.topol.2007.01.018.

[19] E Robinson, Power-Domains, Modalities and the Vietoris Monad, Technical Report 98, Computer Laboratory, University of Cambridge, 1986.

[20] J Rutten, Weighted Colimits and Formal Balls in Generalized Metric Spaces, Topology and its Applications 89 (1998) 179-202; doi:10.1016/S0166-8641(97)00224-1.

[21] D Scott, Lattice Theory, Data Types and Semantics, from: "Formal Semantics of Programming Languages", (R Rustin, editor), Courant Computer Science Symposia 2, Prentice-Hall, Englewood Cliffs, N.J., 1972, 65-106.

[22] M Smyth, Totally Bounded Spaces and Compact Ordered Spaces as Domains of Computation, from: "Topology and Category Theory in Computer Science", (G Reed, A Roscoe, R Wachter, editors), Oxford University Press, 1991, 207-229.

[23] R Street, The Formal Theory of Monads, Journal of Pure and Applied Algebra 2 (1972), 149-168; doi:10.1016/0022-4049(72)90019-9.

[24] P Taylor, A Lambda Calculus for Real Analysis, from: "Computability and Complexity in Analysis", (T Grubba, P Hertline, H Tsuiki, K Weihrauch, editors), Informatik Berichte 326, FernUniversität in Hagen (2005), 227-266. Revised version at http: //www paultaylor.eu/ASD/lamcra/.

[25] S Vickers, Topology via Logic, Cambridge University Press, 1989.

[26] S Vickers, Information Systems for Continuous Posets, Theoretical Computer Science 114 (1993), 201-229; doi:10.1016/0304-3975(93)90072-2.

[27] S Vickers, Locales are Not Pointless, from: "Theory and Formal Methods of Computing 1994”, (C Hankin, I Mackie, R Nagarajan, editors), Imperial College Press, London, 1995, 199-216. 
[28] S Vickers, Constructive Points of Powerlocales, Mathematical Proceedings of the Cambridge Philosophical Society 122 (1997), 207-222; doi:10.1017/S0305004196001636.

[29] S Vickers, Localic Completion of Quasimetric Spaces, Technical Report DoC 97/2, Department of Computing, Imperial College, London, 1998.

[30] S Vickers, Topical Categories of Domains, Mathematical Structures in Computer Science 9 (1999), 569-616; doi:10.1017/S0960129599002741.

[31] S Vickers, Strongly Algebraic $=$ SFP (Topically), Mathematical Structures in Computer Science 11 (2001), 717-742; doi:10.1017/S0960129501003437.

[32] S Vickers, The Double Powerlocale and Exponentiation: A Case Study in Geometric Reasoning, Theory and Applications of Categories 12 (2004), 372-422.

[33] S Vickers, Localic Completion of Generalized Metric Spaces I, Theory and Applications of Categories 14 (2005), 328-356.

[34] S Vickers, Some Constructive Roads to Tychonoff, from: "From Sets and Types to Topology and Analysis: Towards Practicable Foundations for Constructive Mathematics", (L Crosilla, P Schuster, editors), Oxford Logic Guides 48, Oxford University Press, 2005, 223-238.

[35] S Vickers, Compactness in Locales and in Formal Topology, Annals of Pure and Applied Logic 137 (2006), 413-438; doi:10.1016/j.apal.2005.05.028.

[36] S Vickers, Locales and Toposes as Spaces, from: "Handbook of Spatial Logics", (M Aiello, I E Pratt-Hartmann, J F van Benthem, editors), Springer, 2007, 429-496.

[37] S Vickers, Sublocales in Formal Topology, Journal of Symbolic Logic 72 (2007), 463-482; doi:10.2178/js1/1185803619.

[38] S Vickers, The Connected Vietoris Powerlocale, Topology and its Applications 156 (2009), 1886-1910; doi:10.1016/j.topol.2009.03.043.

[39] K R Wicks, Fractals and Hyperspaces, Lecture Notes in Mathematics 1492, SpringerVerlag, 1991.

[40] G Winskell, On Powerdomains and Modality, Theoretical Computer Science 36 (1985), 127-137; doi:10.1016/0304-3975(85)90037-4.

School of Computer Science, University of Birmingham

Birmingham B15 2TT, UK

s.j.vickers@cs.bham.ac.uk

http://www.cs.bham.ac.uk/ sjv

Received: 20 March $2009 \quad$ Revised: 22 September 2009 